\title{
MODULAR CONSTRUCTIONS FOR COMBINATORIAL GEOMETRIES
}

\author{
BY \\ TOM BRYLAWSKI $\left({ }^{1}\right)$
}

\begin{abstract}
R. Stanley, in an investigation of modular flats in geometries (Algebra Universalis 1-2 (1971), 214-217), proved that the characteristic polynomial $\chi(x)$ of a modular flat $x$ divides the characteristic polynomial $\chi(G)$ of a geometry $G$. In this paper we identify the quotient: THEOREM. If $x$ is a modular flat of $G, x(G) / x(x)=\chi\left(\bar{T}_{x}(G)\right) /(\lambda-1)$, where $\bar{T}_{x}(G)$ is the complete Brown truncation of $G$ by $x$. (The lattice of $\bar{T}_{x}(G)$ consists of all flats containing $x$ and all flats disjoint from $x$, with the induced order from $G$.) We give many characterizations of modular flats in terms of their lattice properties as well as by means of a short-circuit axiom and a modular version of the MacLane-Steinitz exchange axiom.

Modular flats are shown to have many of the useful properties of points and distributive flats (separators) in addition to being much more prevalent. The theorem relating the chromatic polynomials of two graphs and the polynomial of their vertex join across a common clique generalizes to geometries: THEOREM. Given geometries $G$ and $H$, if $x$ is a modular flat of $G$ as well as a subgeometry of $H$, then there exists a geometry $P=P_{x}(G, H)$ which is a pushout in the category of injective strong maps and such that $X(P)=$ $\chi(G) \chi(H) / \chi(x)$. The closed set structure, rank function, independent sets, and lattice properties of $\boldsymbol{P}$ are characterized. After proving a modular extension theorem we give applications of our results to Crapo's single element extension theorem, Crapo's join operation, chain groups, unimodular geometries, transversal geometries, and graphs.
\end{abstract}

1. Introduction. The purpose of this paper is to make an extensive study of the concept of modularity in the theory of finite synthetic geometry. We relate the concept of modularity with present trends of research in geometries (most notably with representation of geometries and the critical problem of Crapo and Rota [16]), and we show how the existence of a modular element facilitates the computation of invariants and makes possible certain geometrical constructions while simplifying others.

Presented to the Soceity, January 25, 1973; received by the editors December 27, 1972 and, in revised form, March 15, 1974.

AMS (MOS) subject classifications (1970). Primary 05B25, 05B35; Secondary 05B20, 05C15, 06A30, 15A21, 15A36, 18A30, 50D35, 94A20.

(1) This research was supported in part by NSF Grant GP-32838. 
Historically, modularity has played an important role in the study of combinatorial geometries. In fact the present study of finite geometries rests on the nineteenth century work on projective spaces, where all elements are modular and apparently Birkhoff intended his notion of semimodularity [1] to be a generalization of modularity. It is interesting to note that while the more general idea of a modular pair and the more specialized case of a distributive flat both received more attention in [16] than modular flats, theorems involving modular pairs can be considerably strengthened when one element of the pair is modular and theorems involving distributive flats do not need to be weakened very much to be applied to modular flats. For example, while modular pairs $x$ and $y$ induce an order-preserving monomorphism between the intervals $[x \wedge y, x]$ and $[y, x \vee y]$, the monomorphism becomes an isomorphism when $x$ is modular (3.4.4). On the other hand, Propositions (3.11), (3.17), and (7.2) concerning modularity compare favorably with their distributive counterparts. Further, in the study of special classes of geometries, like graphs, the special properties of modular flats (e.g. cliques) have been noted with interest. Modularity plays an implicit role in much of the current research on geometries. In the ring which Graves associates with a geometry [17], it is precisely the modular elements which are not canceled by their complements.

However, it was not until R. Stanley made the remarkable discovery that the characteristic polynomial of a modular flat divides the characteristic polynomial of the geometry which contains it [22] that interest in modular flats began in earnest. It had been well known that the characteristic polynomial of a geometry was a multiple of the polynomial of any of its distributive flats (separators) as well as of the polynomial $(\lambda-1)$ of a point; Stanley synthesized these two facts and showed that this property which holds for separators and points also holds for modular flats. Our principal motivation arises from this theorem of Stanley-what other properties shared by points and separators also apply to more general modular flats? One is tempted to make an analogy with general topology where some properties of single points extend to compacta.

For example, Crapo in a series of lectures at Bowdoin College [15] suggested as a research problem that one should find conditions sufficient for a pushout to exist in the category of geometries and strong maps. Such a pushout from a subgeometry $x$ to geometries $G$ and $H$ can be trivially constructed when $x$ is a separator of either $G$ or $H$, and it was shown in [6] that pushouts from points also exist. The generalization to modular flats then became the sought for Theorem (5.3). Example 5.4.3 shows that the modularity of $x$ is not necessarv for the existence of a pushout; but it is not surprising that the smallest counterexample showing pushouts do not exist in general, (5.4.1), also involves the simplest example of a nonmodular flat. 
In $\S 2$ we give a survey of the basic definitions and properties of finite combinatorial geometries needed in the remainder of the paper. The reader is encouraged to use this section mainly for reference. A more detailed account of these basic properties [9] can be obtained by request from the author.

In $\S 3$ we explore modularity in various cryptomorphic contexts. After making an extensive study of the behavior of modular elements in a geometric lattice, we show that the structure of the set of all modular elements in a geometry is similar to that of (the order dual of) a geometry. (For example, Greene's results [18] on Whitney number inequalities appear in an analogous form in this context.) Lemmas asserting that modularity is transitive and that pairs of modular elements are closed under infimum are straightforward generalizations of analogous distributivity theorems. We then show that taking minors of geometries preserves modularity. The "modular short-circuit axiom" follows and bears a resemblance on the one hand to the general circuit elimination axiom (when the modular flat in question is a point) and on the other hand to the fact that separators partition the family of circuits. A modular flat is characterized as a subset of a geometry in terms of the geometry's circuits, its independent sets, and its closure operator.

Not only does modularity generalize distributivity, but it also interrelates with it, so that a direct sum decomposition of a modular flat induces a decomposition of the geometry. This implies, for example, that a line in a connected binary geometry is modular if and only if it contains three points. Also, if an upper interval is separable, then its zero element is modular in the subgeometry of one of its separators if and only if the other separator is modular in the geometry.

In $\S 4$ we show how modularity can be characterized within certain special subclasses of geometries-chain groups, unimodular chain groups, graphs, and transversals.

In $\S 5$ we generalize our work on the category of pointed pregeometries [6] and construct the generalized parallel connection of two geometries relative to a common subgeometry which is modular in one of the geometries. This construction can best be characterized in terms of its closed sets: a subset is closed in the generalized parallel connection if and only if its intersection with each geometry is closed. This property is sufficient to ensure the existence of a categorical pushout (direct sum in the category of pairs), but we also give an example of a pushout which does not have this closed set structure and an example of a geometry which has this closed set but is not a generalized parallel connection. The rank function, closure operator, and independent sets of the connection are then described, and we establish several commutativity properties of connections which are smilar to some categorical properties of pushouts. As 
an application of the theory of generalized parallel connections, we prove that a single element extension of a modular flat induces a unique single element extension of the geometry; this generalizes the known result [16] that a pregeometry has a canonically induced geometry. The existence of this unique extension implies that one can tell from the structure of an upper interval whether or not a geometry is a generalized parallel connection. The generalized parallel connection is shown to be a special case of Crapo's join operation [13], [14], [15].

Two other constructions, the Brown truncation [5] and the complete Brown truncation are easily characterized when the truncated flat is modular. Finally, we relate our categorical work to the category of pregeometries and weak maps, and we show that even in this richer category, pushouts do not exist in general.

In $\S 6$ we describe an extension property for various classes of geometries, proving that certain classes (graphs as well as unimodular, binary, and ternary chain groups) have this extension property and some (transversals) do not. We conjecture that all chain groups over finite fields have this property. The extension property guarantees in all the above cases that these classes are closed under generalized parallel connections; we explicitly construct connections within each class. We also suggest a method to extend the graphical notion of multiple connectivity to more general geometries, and we show how the existence of a modular flat affects this connectivity.

In our final section (\$7), we give a new proof of Stanley's theorem on the factorization of the characteristic polynomial of a geometry and provide a geometrical interpretation of the quotient obtained. These results suggest the existence of a more general factorization induced by any flat; we hope to study this problem in a later paper, since any such result would yield added insights into the critical problem of Crapo and Rota [16]. Finally, we show that the characteristic polynomial of a generalized parallel connection is equal to the product of the polynomials associated with its two components divided by the polynomial of their common subgeometry. This theorem is best seen as a generalization of the classical theorem on graph coloring involving the analogous computation of the chromatic polynomial of the vertex join of two graphs across a common clique.

The author wishes to thank the National Science Foundation for allowing him to attend its 1971 summer conference on combinatorial theory, where he originated some of these ideas, and for its grant which gave him the opportunity to pursue them. He would also like to thank Dr. Richard Stanley for his helpful comments, and the referee for pointing out the connections between the present paper and Crapo's work on joins. 
2. Basic definitions. This section surveys the relevant notions of the underlying category for our work, $G$, the category of finite combinatorial geometries and strong maps discussed in Crapo and Rota [16].

A finite pregeometry or matroid, $G$, is a finite set of points with a closure operator $J_{G}\left(\bullet^{\bullet}\right)$ satisfying the exchange property: For any points $p, q \in G$ and any subset $P \subseteq G$, if $p \in \overline{P \cup\{q\}}$ but $p \notin \bar{P}$, then $q \in \overline{P \cup\{p\}}$. When confusion might arise we denote by $\{G\}$ the points of $G$ (without the closure structure). A geometry is a pregeometry in which the empty set and each point is closed. The lattice, $L$, of closed sets or flats of a geometry is called a geometric lattice and is characterized as a finite, semimodular, point lattice. Flats covering 0 in $L$ are called atoms (which for a geometry may be identified with the points) and flats covered by 1 are called coatoms or copoints. Therefore for a flat $x$ and point $p, p \leqslant x$ (in $L$ ) iff $p \in x$ (in $G$ ). A bond $B$ of $G$ is the set complement of a copoint. In such lattices, each lattice element $x$ is the supremum of atoms (points) and each has a well-defined rank, $r(x)$, equal to the length of any maximal chain from the 0 element (representing the empty set) to $x$. The semimodular law for $L$ states that, for all flats $x, y \in L$, $r(x)+r(y) \geqslant r(x \wedge y)+r(x \vee y)$. Flats $x$ and $y$ form a modular pair if the latter inequality is an equality; $x$ is a modular flat if it forms a modular pair with every other flat. $r(A)$, the rank of a set of points $A \subseteq G$, is defined as $r(\bar{A})$ in the associated geometric lattice. Hence $r(G)$, the rank of the geometry, is $r(1)$ in the lattice.

For any subset $A$, the cardinality of $A,|A|$ denotes the number of points it contains; the corank of $A$ is the nonnegative integer $r(G)-r(A)$; and the nullity of $A$ is the nonnegative integer $|A|-r(A)$. A set of points $A \subseteq G$ represents a spanning set for $G$ if $\bar{A}=G$. A set of points $A$ is independent if $r(A)=|A|$. Otherwise, $r(A)<|A|$ and $A$ is dependent. An independent spanning set is called a basis.

A strong map from a geometry $G$ into $H$ is a function $f:\{G\} \cup\{0\} \rightarrow$ $\{H\} \cup\{0\}$ (where " $O$ " stands for the empty set in $G$ and $H$ respectively) such that $f(0)=0$ and the inverse image of any closed set in $H$ is closed in $G$. Geometries $G$ and $H$ are isomorphic, denoted $G \simeq H$, if there is a bijection $f$, taking points of $G$ onto $H$ and closed sets of $G$ onto $H$ such that, for any point $p$ and closed set $K, p \in K$ iff $f(p) \in f(K)$.

A geometry on the point set $\{G\}$ can be uniquely determined by $C(G)$, the family of minimal dependent sets or circuits of $G$. A family $F$ of subsets is the circuit set for some pregeometry if no subset in $F$ properly contains another and the subsets satisfy the circuit elimination property $C^{*}:$ If $C_{1}$ and $C_{2}$ are two distinct elements of $F$ and $p \in C_{1} \cap C_{2}$ then the set difference $\left(C_{1} \cup C_{2}\right)-\{p\}$ is dependent and contains an element $C_{3} \in F$. Further, $C_{3}$ 
can be constructed so as to contain any specified point in $C_{1}-C_{2}$.

$G$ may also be uniquely determined from its set of bases, $B(G)$. A family $F$ of incomparable subsets is the set of bases for some pregeometry if $F$ satisfies the basis exchange axiom $B^{*}$ : For all $B_{1}$ and $B_{2}$ in $F$ and $p \in B_{1}$ there exists $q \in B_{2}$ such that $\left(B_{1}-\{p\}\right) \cup\{q\}$ is also in $F$.

The (Whitney) dual of $G, \widetilde{G}$, is the unique pregeometry on the same point set with a set of bases consisting of base complements of $G$. Hence $B \in$ $B(\widetilde{G})$ iff $\{G\}-B \in B(G)$.

$G$ is the direct sum of two geometries: $G_{1} \oplus G_{2}$ if the points of $G$, $\{G\}$, and circuits of $G, C(G)$, are the disjoint union $\left\{G_{1}\right\} \cup\left\{G_{2}\right\}$ and $C\left(G_{1}\right) \cup C\left(G_{2}\right)$ respectively (equivalently $B(G)$ is the set cartesian product $\left.B\left(G_{1}\right) \times B\left(G_{2}\right)\right) . G_{1}$ is then said to be a direct sum factor of $G$, and $G$ is termed separable with the flats $G_{1}$ and $G_{2}$ in $L$ as separators or distributive flats. If no such nontrivial direct sum decomposition exists, any two distinct points of $G$ are contained in a circuit and $G$ is termed connected. A one point direct sum factor, $p$, is an isthmus and is in no circuits of $G$.

If $p \in G$ we define two derived geometries: the deletion, $G-p$, on the point set $\{G\}-\{p\}$ and the contraction whose geometric lattice is isomorphic to the interval $[p, G]$. If $A \subseteq\{G\}-\{p\}$, and $\bar{A}$ denotes its closure in $G$, then the closure of $A$ in $G-p$ is defined as $\bar{A}-\{p\}$. If $D \subseteq G$, the subgeometry $G-D$ is defined as a sequence of deletions by points in $D$. Similarly we define the contraction $[x, G]$ as a sequence of contractions. An arbitrary sequence of contractions and deletions is called a minor.

An invariant is a function $f$ defined on the class of all pregeometries such that $f(G)=f(H)$ if $G \simeq H$. Examples of invariants used in this paper include $\mu(G)$, the Möbius function which is defined as $\mu(0,1)$ evaluated on the geometric lattice $L$ associated with $G$; the characteristic polynomial,

$$
\chi(G, \lambda)=\chi(G)=\sum_{x \in G} \mu(0, x) \lambda^{r(1)-r(x)} ;
$$

and $\beta(G)$, the Crapo invariant which is explored in [12].

\section{Properties of modular flats.}

DEFINITION 3.1. In a geometry $G$, two flats $x$ and $y$ form a modular pair denoted $M(x, y)$ if $r(x)+r(y)=r(x \wedge y)+r(x \vee y)$ where $r$ is the rank function of the associated geometric lattice. Note that modularity so defined is a symmetric relation. Indeed it is the fact that this relation is symmetric and corresponds to the general lattice theoretic definition (3.2.2) for semimodular lattices, which is a principal result of [26]. A flat $x$ is modular if $M(x, y)$ for all flats $y$ of $G$.

Examples of modular flats of $G$ include $G$, the empty set, any point, 
and any separator. In fact we occasionally call a separator a distributive flat since it satisfies the distributive law $(u \vee y) \wedge x=(u \wedge x) \vee(y \wedge x)$ for all flats $u$ whereas a modular flat satisfies this law for all $u \leqslant x$ (3.3.2). A geometry is modular if all its flats are modular iff the geometry is the direct sum of (perhaps degenerate) projective spaces [1].

LEMmA 3.2. The following are equivalent for flats $x$ and $y$ in a geometric lattice.

1. $M(x, y)$.

2. $(u \vee y) \wedge x=u \vee(y \wedge x)$ for all flats $u \leqslant x$.

3. $y$ is a minimal relative complement of $x$ in the interval $[x \wedge y, x \vee y]$.

Proof. This is Proposition (2.8) in [16].

THEOREM 3.3. The following are equivalent for a flat $x$ in a geometric lattice:

1. $x$ is modular.

1'. For all flats $y$ disjoint from $x, r(y)+r(x)=r(x \vee y)$.

1". For all complements $y$ of $x, r(x)+r(y)=r(1)$.

2. For all flats $u \leqslant x$ and all flats $y$ (equivalently, 2', all complements $y$ of $x),(u \vee y) \wedge x=u \vee(y \wedge x)$.

3. For all pairs of flats $y \leqslant v$ (equivalently, 3', for all complements $v$ of $x$ and $y \leqslant v),(y \vee x) \wedge v=y \vee(x \wedge v)$.

4. For all flats $y$ (equivalently, 4', for all complements $y$ of $x$ ), $[x \wedge y, x] \simeq[y, x \vee y]$ (by the order homomorphism $f(u)=u \vee y$ with inverse $\left.f^{-1}(v)=v \wedge x\right)$.

5. For all flats $y$ of $G, y$ is a minimal relative complement of $\boldsymbol{x}$ (equivalently, $x$ is a minimal relative complement of $y$ ) in the interval $[x \wedge y, x \vee y]$.

5'. All complements of $x$ are incomparable.

6. The flat $x$ can never be $v$ (equivalently, $w$ ) in a sublattice of the form:

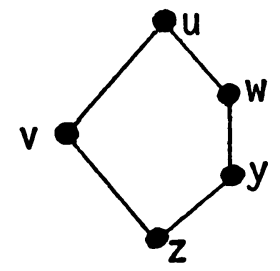

7. For all flats $y$ and points $p \in x,(p \vee y) \wedge x=p \vee(y \wedge x)$.

8. For all $p \notin x, x \simeq[p, x \vee p]$ (by the inverse order homomorphisms $f(u)=u \vee p$ and $\left.f^{-1}(v)=v \wedge x\right)$ and further $x \vee p$ is modular in the contraction $[p, 1]$. 
Proof. $(1) \Leftrightarrow\left(1^{\prime}\right) \Leftrightarrow\left(1^{\prime \prime}\right)$. Clearly $(1) \Rightarrow\left(1^{\prime}\right) \Rightarrow\left(1^{\prime \prime}\right)$. To show $\left(1^{\prime \prime}\right) \Rightarrow$ (1), let $y$ be any flat of $G$. Further, let $w$ be a minimal (modular) relative complement of $x \vee y$ in the interval $[y, 1]$; let $z$ be a minimal relative complement of $x \wedge y$ in the interval $[0, y]$; and let $x^{\prime}$ be a minimal relative complement of $y$ in the interval $[z, w]$. Referring to the following diagram:

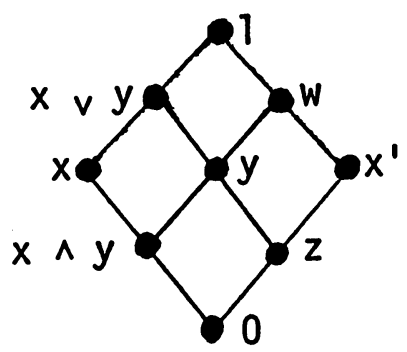

we have $x \wedge x^{\prime} \leqslant(x \vee y) \wedge w=y$ so that $x \wedge x^{\prime} \leqslant y \wedge x^{\prime}=z$, and $x \wedge x^{\prime}$ $\leqslant x \wedge y$. Therefore $x \wedge x^{\prime} \leqslant z \wedge(x \wedge y)=0$. Similarly $x \vee x^{\prime}=1$. Thus $x^{\prime}$ is a complement for $x$. But whenever $u$ is a minimal relative complement of $v$,

$$
r(u \vee v)-r(u)=r(v)-r(v \wedge u)
$$

We thus obtain the equalities:

$$
r(1)-r(x \vee y)=r(w)-r(y)=r\left(x^{\prime}\right)-r(z) .
$$

By hypothesis, $r(1)-r(x)=r\left(x^{\prime}\right)$, so that

$$
\begin{aligned}
r(x \vee y)-r(x) & =(r(1)-r(x))-(r(1)-r(x \vee y)) \\
& =r\left(x^{\prime}\right)-\left(r\left(x^{\prime}\right)-r(z)\right)=r(z)=r(y)-r(x \wedge y) .
\end{aligned}
$$

$(1) \Rightarrow(2) \Rightarrow\left(2^{\prime}\right) \Rightarrow\left(1^{\prime \prime}\right)$. By (3.2), (1) and (2) are equivalent to $M(x, y)$ for all flats $y$; while $\left(2^{\prime}\right)$ and $\left(1^{\prime \prime}\right)$ are equivalent to $M(x, y)$ for all complements $y$ of $x$.

$(1) \Rightarrow(3) \Rightarrow\left(3^{\prime}\right) \Rightarrow\left(1^{\prime \prime}\right)$. Similar to above using $M(v, x)$.

(4) $\Rightarrow$ (1). Trivial.

(2), (3) $\Rightarrow(4)$. By (3) and the fact that $y \leqslant v \leqslant y \vee x, f\left(f^{-1}(v)\right)=$ $y \vee(x \wedge v)=(y \vee x) \wedge v=v$. By (2) and the fact that $x \wedge y \leqslant u \leqslant x$, $f^{-1}(f(u))=(u \vee y) \wedge x=u \vee(y \wedge x)=u$.

$(1) \Leftrightarrow(5)$. By (3.2) both are equivalent to $M(x, y)$ (or $M(y, x)$ ) for all y. Similarly, $\left(1^{\prime \prime}\right) \Leftrightarrow\left(5^{\prime}\right)$.

$(5) \Leftrightarrow(6)$. (6) does not hold iff, in some interval $[z, u], v$ and $w$ are not minimal relative complements.

$(2) \Leftrightarrow(7)$. Clearly (2) $\Rightarrow(7)$. Conversely, if $u \leqslant x$, we will prove (2) by induction on the rank of $u$. If $r(u)=1, u=p \in x$ and (7) holds. Assume we 
have proved (2) for all flats $u^{\prime} \leqslant x$ of rank $k \geqslant 1$. Let $\left\{p_{1}, \cdots, p_{k+1}\right\}$ be a basis for $u$, and let $u^{\prime}=p_{2} \vee \ldots \vee p_{k+1}$. Then $u^{\prime} \leqslant x$ and $r\left(u^{\prime}\right)=k$. Hence, using induction, we have

$$
\begin{aligned}
(u \vee y) \wedge x & =\left(p_{1} \vee \cdots \vee p_{k+1} \vee y\right) \wedge x=\left(p_{1} \vee\left(u^{\prime} \vee y\right)\right) \wedge x \\
& =p_{1} \vee\left(\left(u^{\prime} \vee y\right) \wedge x\right)=p_{1} \vee\left(u^{\prime} \vee(y \wedge x)\right) \\
& =\left(p_{1} \vee u^{\prime}\right) \vee(y \wedge x)=u \vee(y \wedge x)
\end{aligned}
$$

$(8) \Rightarrow\left(1^{\prime}\right)$. If $y \neq 0$ is any flat disjoint from $x$, then there is some $p \notin x$ such that $y \in[p, 1]$. Hence $r(x \vee p)=r(x)+1 ;(x \vee p) \vee y=$ $x \vee y$; and $(x \vee p) \wedge y=p$ since by the isomorphism in (8), $f^{-1}((x \vee p) \wedge y)$ $=(x \vee p) \wedge y \wedge x=x \wedge y=0$, so that $(x \vee p) \wedge y=f(0)=p$. Using the modularity of $x \vee p$ in the second equality:

$$
\begin{aligned}
r(x)+r(y) & =r(x \vee p)+r(y)-1=r((x \vee p) \vee y)+r((x \vee p) \wedge y)-1 \\
& =r(x \vee y)+r(p)-1=r(x \vee y) .
\end{aligned}
$$

$\left(1^{\prime}\right),(4) \Rightarrow(8)$. By (4), $x \simeq[p, x \vee p]$. We will show that $x \vee p$ forms a modular pair with any relatively disjoint flat $y$ in $[p, 1]$ (i.e. any flat $y$ such that $y \wedge(x \vee p)=p)$, which will then show $x \vee p$ is modular in $[p, 1]$ by (1'). But if $y \wedge(x \vee p)=p$ then $y \wedge x=p \wedge x=0$; so if $r^{\prime \prime}$ denotes the induced rank function in $[p, 1]$,

$$
r^{\prime \prime}(x \vee p)+r^{\prime \prime}(y)=r(x)+r(y)-1=r(x \vee y)-1=r^{\prime \prime}(x \vee y) \text {. }
$$

Special cases of the various conditions in (3.3) abound. For example, the following is a corollary of (3.3.1').

COROLLARY 3.4. A hyperplane is modular if and only if it intersects every line.

The following two propositions were suggested by R. Stanley. In fact (3.6) appears in [22].

Proposition 3.5. If $x$ is modular in $G$ and $u$ is modular in the closed subgeometry $x$, then $u$ is modular in $G$.

Proof. Let $u, u^{\prime}$ be complements in $G$. Then since $x$ is modular in $G$ and $x \geqslant u$,

$$
r(x)+r\left(u^{\prime}\right)=r\left(x \vee u^{\prime}\right)+r\left(x \wedge u^{\prime}\right)=r(1)+r\left(x \wedge u^{\prime}\right) .
$$

Further, since $u$ is modular in $x$,

$$
r(u)+r\left(x \wedge u^{\prime}\right)=r\left(u \vee\left(x \wedge u^{\prime}\right)\right)+r\left(u \wedge x \wedge u^{\prime}\right) \text {. }
$$

But $u \wedge x \wedge u^{\prime}=u \wedge u^{\prime}=0$; and, by the modularity of $x, u \vee\left(x \wedge u^{\prime}\right)=$ 
$\left(u \vee u^{\prime}\right) \wedge x=x$. Therefore $r(x)=r(u)+r\left(x \wedge u^{\prime}\right)$ and $r(u)+r\left(u^{\prime}\right)=r(u)+$ $\left(r(1)-r(x)+r\left(x \wedge u^{\prime}\right)\right)=r(1)$ so we are done by (3.3.1").

PROPOSITION 3.6. If $x$ and $z$ are modular flats, so is $x \wedge z$.

Proof. Noting that if $u \leqslant x \wedge z$ then $u \leqslant x$ and $u \leqslant z$. If we use (3.3.2) first for the modular flat $x$ and then for $z$, we get for any flat $y$,

$$
\begin{aligned}
(u \vee y) \wedge(x \wedge z) & =((u \vee y) \wedge x) \wedge z=(u \vee(y \wedge x)) \wedge z \\
& =u \vee((y \wedge x) \wedge z)=u \vee(y \wedge(x \wedge z))
\end{aligned}
$$

We mention in passing that many of our results yield as corollaries characterizations of projective geometries. For example, a geometry is modular iff every hyperplane is modular (3.6) iff every hyperplane meets every line (3.4) iff every line is modular (3.3.1") iff for some $k \in[2, n-1]$ every flat of rank $k$ is modular (3.6) iff for some $k \in[2, n-1]$ every flat of rank $k$ meets every flat of rank $n-k+1$ (3.3.1"), a result of [27].

The following theorem suggests similarities with Greene's work on inequalities of Whitney numbers [18] as well as unexplored connections with Dilworth completions of lower truncated geometries [16].

THEOREM 3.7. In a geometry $G$ :

1. The order dual $L^{*}$ of the infimum subsemilattice $L$ generated by the modular copoints forms a geometric lattice.

2. The number of modular copoints is less than or equal to the number of atoms with equality if and only if $G$ is modular.

The following two statements hold for the case when $G$ contains a modular copoint. For a more general geometry we may get analogous results by upper truncating the geometric lattice $L$ of $G$ to level $k$ if $G$ has a modular flat of maximal rank $k-1$.

3. The subset of modular elements $M$ of $G$ under the induced ordering forms a geometric lattice if and only if $G=M$.

4. $M^{*}$ is a geometric lattice if and only if all the modular elements are infima of modular copoints.

Proof. (1) Clearly $L^{*}$ as constructed is a point lattice and since $L$ is formed by taking infima of modular copoints, if the rank of $G$ is $n$ then $r^{*}(x)$, the rank of an element $x$ in $L^{*}$, is given by $n-r(x)$. Further, by (3.6) all the elements of $L$ are modular in $G$. Hence

$$
\begin{aligned}
r^{*}(x)+r^{*}(y) & =n-r(x)+n-r(y)=n-r(x \wedge y)+n-r(x \vee y) \\
& =r^{*}\left(x \vee^{*} y\right)+n-r(x \vee y),
\end{aligned}
$$


where $\mathrm{V}^{*}$ is the supremum operator in $L^{*}$. But in $G$ the $L^{*}$ infimum of $x$ and $y, x \wedge^{*} y$, is greater than or equal to $x \vee y$ (since this is the infimum in $G^{*}$ ). Hence $n-r(x \vee y) \geqslant r^{*}\left(x \wedge^{*} y\right)$ and $L^{*}$ is semimodular. Thus $L^{*}$ is geometric.

(2) If the 0 element of $L$ constructed in (1) is $x$, then $f(p)=p \vee x$ is a surjection in $G$ from the points $P$ of $G$ onto the atoms $A$ of $L$. But $A$ is the set of copoints of $L^{*}$ while the points of $L^{*}$ are the modular copoints $C$ of $G$. Since (1) shows $L^{*}$ is geometric and in [18] it is shown that in a geometric lattice the number of copoints is greater than or equal to the number of points, we have $|P| \geqslant|A| \geqslant|C|$. Further, $|P|>|A|$ unless $x=O_{G}$ and all the atoms are infima of modular copoints. Further (again by [18]) $|A|>|C|$ unless $L^{*}$ (and hence $L$ ) is modular. But in that case $L$ is geometric (it is surely a point lattice) and there is a rank preserving strong map from $L$ into $G$ which is bijective on points. Thus, by $(9.15)$ of $[16], L \simeq G$.

(3) Since all the points are modular, (3) follows from (2) along with the inequality on Whitney numbers of [18].

(4) As in the proof of (1), $M^{*}$ will be semimodular and so it will be geometric if and only if it is a point lattice.

The following proposition parallels (3.3.8) for deletion, the dual operation of contraction.

PROPOSITION 3.8. If $x$ is a modular flat of $G$ and $p \notin x$ then $x$ as a subgeometry of $G-p$ is modular in the deletion and isomorphic to $x$ as a subgeometry of $G$. Hence $x$ is a modular flat of any subgeometry $G$ which contains it.

PROoF. The rank function $r^{\prime}$ on subsets of a deletion (or subgeometry) is the same as the rank function $r$ on those subsets in $G$. Hence $x$ is closed and isomorphic to itself as a subgeometry both of $G$ and $G-p$. Furthermore, for any flat $y$ disjoint from $x$ in $G-p, \bar{y}=y$ or $\bar{y}=y \cup p$ in $G$ and in any case is disjoint from $x$. Hence,

$$
\begin{aligned}
r^{\prime}(x)+r^{\prime}(y) & =r(x)+r(y)=r(x)+r(\bar{y})=r(x \vee \bar{y}) \\
& =r(x \cup \bar{y})=r(x \cup y)=r^{\prime}(x \cup y)=r^{\prime}(x \vee y) .
\end{aligned}
$$

We are then done by (3.3.1').

COROLlaRY 3.9. If $x$ is a modular flat of $G$, then $x \vee y$ as a subminor of any minor $[y, G-A]$ where $(A \cup y) \cap x=\varnothing$ is modular and isomorphic to $x$ as a subgeometry of $G$. Further, if $y \leqslant x$, then $[y, x]$ is modular in the contraction $[y, G]$.

Proof. The first statement follows from (3.3.8) and (3.8), while the second is true since if (3.3.6) holds in $G$ it holds in any interval.

REMARKS 3.10. A set of points forms a distributive flat (separator) if and 
only if it contains all the circuits it intersects. More generally we will give a circuit characterization of modular flats.

An alternate proof of the following theorem (3.11) can be given using the fact that whenever $x$ is a flat of a geometry $G$, and $p \in G-x$, then $x \simeq$ $[p, x \vee p]$ by the strong map $f(u)=u \vee p$ if and only if for all circuits $C$ such that $p \in C$ and $|C-x|=2$, the line $\overline{C-x}$ intersects $x$. All details are left to the reader.

TheOREM 3.11 (The modular short-circuit axiom). A nonempty set of points $S$ forms a modular flat of a geometry $G$ if and only if for every circuit $C$ of $G$ and $p \in C-S$ there is $a q \in S$ and circuit $C^{\prime}$ such that $p \in C^{\prime} \subseteq$ $q \cup(C-S)$ (or, equivalently, that $q \cup(C-S)$ is dependent).

PRoof. We first show that the final two conditions are equivalent. Clearly if $p \in C^{\prime} \subseteq q \cup(C-S)$, then $q \cup(C-S)$ is dependent. For the converse we use induction on $|C-S|$. If $|C-S|=1$ they are obviously equivalent. Assume they are equivalent for all circuits $C$ such that $|C-S|<k$ and let $|C-S|=k \geqslant 2$. Then if $q \cup(C-S)$ is dependent it must contain a circuit $C^{\prime}$. If $p \in C^{\prime}$, we are done. Otherwise we use the circuit elimination axiom with circuits $C$ and $C^{\prime}$ relative to points $p \in C-C^{\prime}$ and $q^{\prime} \in C^{\prime}-S \subseteq C^{\prime}$ $\cup C$ (which is nonempty since $C^{\prime} \subseteq S$ would contradict the minimality of $C$ ). Hence there is a circuit $C^{\prime \prime}$ contained in $C \cup C^{\prime}-q^{\prime}$ with $p \in C^{\prime \prime}$. But $C^{\prime \prime}-S$ contains $C-S$ strictly and we are done by induction.

Under the conditions of the theorem, $S$ must be a flat since it cannot contain a broken circuit as it would then contain a one element broken circuit which never happens in a geometry.

Let $S$ be a modular flat and let $C$ be a circuit of cardinality $k$. If $|C \cap S|=0$ or 1 or $|C-S|=0$ there is nothing to prove, so assume $|C \cap S|=k_{1}>1$ and $\left.\mid C \cap S\right]=k_{2}>0$ and both sets are independent. Then

$$
\begin{aligned}
k & =k_{1}+k_{2}=r(\overline{C \cap S})+r(\overline{C-S})=r(\overline{C \cap S} \vee \overline{C-S})+r(\overline{C \cap S} \wedge \overline{C-S}) \\
& =r((C \cap S) \cup(C-S))+r(\overline{C \cap S} \cap \overline{C-S})=k-1+r(\overline{C \cap S} \cap \overline{C-S}) .
\end{aligned}
$$

But then $r(\overline{C \cap S} \cap \overline{C-S})=1$ so that $\overline{C-S}$ must intersect $S$ in a point $q$.

Conversely, assume $S$ is a flat $x$ which is not modular. Hence by (3.3.1') there is a flat $y$ disjoint from $S$ such that $r(x \vee y)<r(x)+r(y)$. Let $S^{\prime}$ be a basis (maximal independent subset) of $S$ and $A$ be a basis for $y$. Then $r\left(A \cup S^{\prime}\right)<|A|+\left|S^{\prime}\right|=\left|A \cup S^{\prime}\right|$ so it contains a circuit $C$ which must intersect both $A$ and $S^{\prime}$. But if $C$ were short-circuited in $S$ we would arrive at the contradiction $q \in \overline{C-S} \cap S \subseteq \bar{A} \cap S=x \wedge y=\varnothing$. 
Definition 3.12. A comodular set of points $S$ of a pregeometry $G$ is one such that $S$ is a modular flat of the Whitney dual $\widetilde{G}$.

COROLlaRY 3.13. If the Whitney dual $\widetilde{G}$ happens to be a geometry, then $S \subseteq G$ is a comodular set if and only if for every copoint $c$ which does not contain $G-S$, there is a copoint $c^{\prime}$ such that $c^{\prime}-S \supseteq c-S$ and $\left|S-c^{\prime}\right| \leqslant 1$.

Proof. This follows from (3.11) and the fact that copoints of $G$ are circuit complements in $\widetilde{G}$.

Corollary 3.14. A two point set $\{p, q\}$ is a modular flat of a geometry $G$ if and only if $p$ and $q$ are in different direct sum components of $G$.

Proof. If points $p$ and $q$ are in the same direct sum component of $G$ then there is a circuit $C$ containing both of them (which, since $G$ is a geometry must contain another point $p^{\prime}$ ). But if $S=\{p, q\}$ were a modular flat then $p^{\prime}$ would be contained in a circuit contained in $(C-\{p, q\}) \cup q^{\prime}$ for some $q^{\prime} \in S$ by (3.11). But this contradicts the minimality of $C$. Conversely, if $p$ and $q$ are in different direct sum components, no circuit can contain both of them and (3.11) is satisfied trivially.

COROLlARY 3.15. In a connected binary geometry $G$ (see 4.1), a line is modular if and only if it contains three points.

Proof. There are no lines of four or more points; two point lines are excluded by (3.14); and three point lines are modular by (3.8) since $G$ is a subgeometry of a binary projective geometry (all of whose lines have three points).

COROLlary 3.16. A separable flat $x$ is modular if and only if each component $x_{i}$ is modular in a separate component $G_{i}$ of $G$. In particular, modular flats of connected geometries are connected.

Proof. The circuit set of $G, C(G)$ is the disjoint union of the circuit sets $C_{i}(G)$ of each component. Hence (3.11) is satisfied in $G$ for $x$ if and only if it is satisfied for $x \cap G_{i}$ in $G_{i}$ for all $i$. Hence $x$ is modular in $G$ if and only if $x \cap G_{i}$ is modular in $G_{i}$ for all direct sum components $G_{i}$. Since a connected subgeometry $S$ must be in a single connected component of $G$ we will be done when we show that in a connected geometry, every modular flat must be connected. But if $x_{1} \oplus x_{2}$ is a separable modular flat of a connected geometry $G$, with $p_{1} \in x_{1}$ and $p_{2} \in x_{2}$, then, by (3.14), $\left\{p_{1}, p_{2}\right\}$ is a modular flat of $x_{1} \oplus x_{2}$ and hence by (3.5) is a modular flat of $G$, contradicting (3.14).

TheOREM 3.17 (The modular exchange axiom). A subset $S$ of a 
geometry $G$ is a modular flat if and only if for all subsets $A \subseteq G$ and points $p$, if $p \in \overline{A \cup S}$ but $p \notin \bar{A}$ then there is a $q \in S$ such that $q \in \overline{A \cup p}(-\bar{A})$.

Proof. If $p \in \overline{A \cup S}-\bar{A}$ then $p$ completes a broken circuit $B$ with elements from $A$ and $S$ (where not all elements are from $A$ ). But if $S$ is a modular flat, by (3.11) there is a circuit consisting of $p$, other elements of $A-S$, and one element $q$ of $S$, so that $q \in \overline{A \cup p}$. Further, $q \notin \bar{A}$ since otherwise we could eliminate $q$ and obtain that $p$ would be in a circuit contained in $A \cup p$.

Conversely, assume $S$ obeys the modular exchange axiom. Let $C$ be a circuit of $G$ which intersects $S$ and let $p \in C-S$. Then, if $A=(C-S)-$ $p, p \in \overline{A \cup S}$ since it completes the broken circuit $C-p$. But $p \notin \bar{A}$ since $A \cup p$ is a proper subset of $C$ and hence is independent. Therefore by the modular exchange axiom there is a $q \in S$ such that $q \in \overline{A \cup p}$. But this means $q$ completes a broken circuit $B^{\prime}$ contained in $A \cup p=C-S$ so that $q \cup$ $(C-S)$ is dependent and $S$ is a modular flat by (3.11).

We have seen how modularity can be characterized directly by the rank function, circuits, and the closure operator. Of course any family of subsets which gives a cryptomorphic characterization of a geometry may be used in turn to distinguish its modular elements. For example, an easy corollary of (3.11) gives a characterization in terms of independent sets:

Proposition 3.18. A set $S$ is modular if and only if for all independent subsets $I \subseteq G-S, I \cup p$ is independent for all $p \in S$ if and only if $I \cup I^{\prime}$ is independent for all independent subsets $I^{\prime}$ of $S$.

We conclude this section with a proposition relating modularity with distributivity.

PROPOSITION 3.19. In a geometry $G$ if the interval $[x, 1]$ is separable with separators $z$ and $y$, then $z$ is a modular flat of $G$ if and only if $x$ is a modular flat of the subgeometry $y$.

Proof. Since $z$ and $y$ are separators of $[x, 1]$, the subsets $x, z-x$, and $y-x$ partition the points of $G$. Hence $u \wedge z=0$ iff $u \subseteq y-x$ iff $u \leqslant y$ and $u \wedge x=0$. Further, if $u \leqslant y$ and $u \vee z=1$ then

$$
\begin{array}{rlrl}
y & =y \wedge(u \vee z)=u \vee(y \wedge z) & \text { (since } y \text { and } z \text { are separators of } \\
& {[x, 1] \text { and hence a modular pair) }}
\end{array}
$$

Conversely if $u \vee x=y$ then $u \vee z=u \vee(x \vee z)=(u \vee x) \vee z=y \vee z=1$. Hence $u$ is a complement of $z$ iff it is a relative complement of $x$ in the subgeometey $y$, and the proposition follows from $\left(3.3 .5^{\prime}\right)$. 
4. Modularity for classes of geometries. In the previous section we characterized modularity for geometries in general. For a geometry with a particular representation (i.e. as a matrix, totally unimodular matrix, graph, or transversal), modular flats can be characterized within the class as subsets with certain properties. In $\S 6$ we will exploit some of these properties to make constructions in the above classes.

\subsection{Chain group (dependence) geometries.}

DEFINITION 4.1.1. A geometry $G$ is a chain group or dependence geometry over a field $F$ if it can be represented as the columns of matrix $M$ (denoted $G=D(M)$ ) with dependence in $G$ corresponding to linear dependence (over $F$ ) of column vectors of $M$. We also say $G$ is coordinatizable over $F$. If $G$ can be coordinatized over the two element field $F_{2}, G$ is termed binary, and if $G$ can be coordinatized over $F_{3}, G$ is termed ternary.

Clearly elementary row operations, and permutation and nonzero scalar multiplication of columns leave the coordinatization invariant and we may delete any zero rows. Hence we may assume our matrix is of size $r \times n$ where $|G|=n$ and $r(G)=r$, and that $r$ of the columns (corresponding to any basis of the geometry) form the identity submatrix.

Proposition 4.1.2. A subset of columns $\left\{v_{1}, \cdots, v_{k}\right\}$ of a chain group $D(M)$ forms a modular flat $x$ if and only if whenever $\left\{v_{k+1}, \cdots, v_{m}\right\}$ independently extend $x$ to a spanning set for $G$ giving the coordinatization

$$
\left[\begin{array}{ccc}
M_{x} & 0 & M_{1} \\
0 & I & M_{2}
\end{array}\right] \quad \text { (where } M_{x}^{\prime}=\left[\begin{array}{c}
M_{x} \\
0
\end{array}\right] \text { represents the flat } x \text {, }
$$

and $I$ is the $(m-k) \times(m-k)$ identity matrix $)$

then each column of $M_{1}$ is a scalar multiple of some column of $M_{x}$.

Proof. By (3.11) $x$ is modular if and only if for any minimally dependent set of vectors $C$ with $v \in C^{-}-M_{x}^{\prime}$ there exists $v^{\prime} \in M_{x}^{\prime}$ such that $v$ is linearly dependent on $v^{\prime} \cup\left(C-M_{x}^{\prime}\right)-v$.

Let $C$ be a basic circuit relative to some basis for $M_{x}^{\prime}$ along with $\left\{v_{k+1}, \cdots, v_{m}\right\}$, so we may assume

$$
C=\left\{v_{1}, \cdots, v_{i} ; v_{k+1}, \cdots, v_{j} ; v\right\} \quad(i \leqslant k, j \leqslant m) .
$$

Then $v \cap M_{2}$ must have nonzero entries only where $v_{k+1}, \cdots, v_{j}$ have nonzero entries. Hence $v$ will complete a circuit with exactly one element from $M_{x}^{\prime}$ along with vectors from $\left\{v_{k+1}, \cdots, v_{j}\right\}$ iff $v \cap M_{1}$ is a nonzero scalar multiple of a column of $M_{x}$ and will complete a circuit disjoint from $M_{x}^{\prime}$ iff 
$v \cap M_{1}=0$. Hence the condition of the proposition for a particular choice of $\left\{v_{k+1}, \cdots, v_{m}\right\}$ is equivalent to (3.11) for any basic circuit relative to a basis containing $\left\{v_{k+1}, \cdots, v_{m}\right\}$. But for any circuit $C$ of $G$ and $v \in C-M_{x}^{\prime}$, $C-v$ is independent and can be extended to such a basis.

REMARKs 4.1.3. To get an idea of the above proposition we observe that the conditions hold readily for the flats $\varnothing, G$, separators (in which case $M_{1}=$ 0 ), and points (where $M_{x}$ is a one by one submatrix).

Also, note the way the proposition is quantified (i.e. the condition must hold for all $\left\{v_{k+1}, \cdots v_{m}\right\}$ which extend a maximal independent subset of $M_{x}^{\prime}$ to a basis) so that for example

$$
\begin{aligned}
& \begin{array}{llllll}
a & b & c & d & e & f
\end{array} \\
& {\left[\begin{array}{lll|l|ll}
1 & 0 & 1 & 0 & 1 & 0 \\
0 & 1 & 1 & 0 & 0 & 1 \\
\hline 0 & 0 & 0 & 1 & 1 & 1
\end{array}\right]}
\end{aligned}
$$

which coordinatizes the geometry

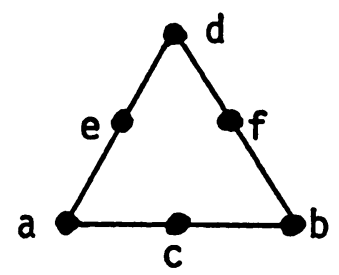

over any field of characteristic not equal to two obeys the conditions of the proposition for $x=\{a, b, c\}$ and basis extension $d$. However $\{a, b, c\}$ is not modular.

\subsection{Unimodular geometries.}

DEFINITION 4.2.1. A geometry $G$ is unimodular if it can be coordinatized over any field. Tutte showed [24] that $G$ is unimodular if and only if it can be coordinatized over the rationals by a totally unimodular matrix $M$ (a matrix all of whose subdeterminants are $0,-1$, or 1 ) in which case $M$ serves to coordinatize $G$ over any field. A geometry so coordinatizable by a totally unimodular matrix is called a unimodular chain group.

LEMMA 4.2.2. Let $M$ be a totally unimodular matrix which coordinatizes a geometry $G$, let $B$ be a basic set of column vectors of $M$, and let $M^{\prime}$ be a matrix which is obtained by performing elementary row reductions to $M$ to get the identity submatrix for its columns $B$. Then $M^{\prime}$ is also totally unimodular and coordinatizes $G$. 
Proof. Elementary row operations cannot change the dependency of the columns so that both $M^{\prime}$ and $M$ coordinatize $G$. Also, permutation and scalar multiplication (by -1 ) of rows cannot change the property of total unimodularity. We will be done (by induction) if we can show that $M^{\prime}$ remains totally unimodular after a sequence of elementary row operations consisting of replacing $r_{j}$ by $a_{j} r_{i}+r_{j}\left(a_{j}=0,1,-1 ; i \neq j\right)$ which transform the column $v_{k}$ into a column such that $m_{i k}= \pm 1$ and $m_{j k}=0$ for all $j \neq i$.

But if $D^{\prime}$ is any square submatrix of $M^{\prime}$ intersecting row $i$ then the row operations cannot change the value of the determinant of $D^{\prime}$ and hence it is 0,1 , or -1 . If $D^{\prime}$ does not intersect row $i$ and intersects column $k$ then its determinant is zero (it has a zero column where it intersects column $k$ ). Finally, if $D^{\prime}$ intersects neither row $i$ nor column $k$, we augment it by adding that row and column forming $D^{\prime \prime}$. Then as above, $D^{\prime \prime}$ has the same determinant in $M$ as $M^{\prime}$. But expanding the determinant of $D^{\prime \prime}$ by cofactors relative to column $k$ we get $\operatorname{det}\left(D^{\prime \prime}\right)= \pm m_{i k} \operatorname{det}\left(D^{\prime}\right)= \pm \operatorname{det}\left(D^{\prime}\right)=0,1$, or -1 .

Proposition 4.2.3. A subset of columns $\left\{v_{1}, \cdots, v_{k}\right\}$ of a unimodular chain group $U(M)$ forms a modular flat $x$ if and only if whenever $\left\{v_{k+1}\right.$, $\left.\cdots, v_{m}\right\}$ extends a basis for $x$ (unimodularly) to a basis for $G$ giving the coordinatization

$$
\left[\begin{array}{lll}
M_{x} & 0 & M_{1} \\
0 & I & M_{2}
\end{array}\right]
$$

then each column of $M_{1}$ is zero or \pm some column of $M_{x}$.

Proof. This follows directly from (4.1.2) and (4.2.2).

4.3. Graphic geometries.

DEFINITION 4.3.1. A geometry is graphic if its point-circuit incidence relation is identical to the edge-circuit (edge-polygon) incidence relation of a graph (the graph must then be of the "Michigan" type with no loops or multiple edges). Hence the geometry is separable if and only if the graph is disconnected or one-connected (with a cut point). Every graph gives such a geometry, while two graphs give isomorphic geometries if and only if they are two-isomorphic [25], i.e. one can be transformed into the other by separating and/or joining sets of edges at a cut point; or by separating two sets of edges with two vertices in common (a parallel connection) and reattaching the sets in the other order. We use the notation $e \sim\left[v, v^{\prime}\right]$ to signify that edge $e$ has vertices $v$ and $v^{\prime}$.

The following characterization is implicitly equivalent to the unproved Theorem 3 of [22]. 
Proposition 4.3.2. A connected subset of edges $E$ of a graph $G$ forms a modular flat if and only if, for every pair of distinct vertices $v, v^{\prime}$ such that there is a path between $v$ and $v^{\prime}$ in $E$ and another path between $v$ and $v^{\prime}$ which is vertex disjoint from $E$ (except for $v$ and $v^{\prime}$ ) then there is an edge $e \in E$ such that $e \sim\left[v, v^{\prime}\right]$.

In particular every clique (i.e. complete subgraph) is modular.

Proof. Assume $E$ forms a modular flat and vertices $v$ and $v^{\prime}$ are such that there is a path $P$ between $v$ and $v^{\prime}$ in $E$ and another path $P^{\prime}$ between $v$ and $v^{\prime}$ which is vertex disjoint from $E$ (except for $v$ and $v^{\prime}$ ). Then $P U$ $P^{\prime}$ forms a circuit and by (3.11) $P^{\prime} \cup e$ must contain a circuit for some $e \in$ $E$. Clearly this can only happen if $e \sim\left[v, v^{\prime}\right]$.

Conversely if $E$ has the above property and $C$ is a circuit intersecting both $E$ and $G-E$, let $P^{\prime}$ be a path contained in $C$ which is vertex disjoint from $E$ (except for its end points $v$ and $v^{\prime}$ ). Then since $E$ is connected, there is a path $P \subseteq E$ from $v$ to $v^{\prime}$. Hence $P \cup P^{\prime}$ is a circuit. Therefore $E$ contains an edge $e \sim\left[v, v^{\prime}\right]$ and $(C-E) \cup\{e\}$ contains $P^{\prime} \cup\{e\}$ which is a circuit.

Thus (3.11) is satisfied.

4.4. Transversal geometries. A transversal geometry $G(S)$ is one presented by a relation $R \subseteq S \times T$ (where every element of $S$ is related to at least one element of $T$ and every pair is related to at least two elements). Such geometries are characterized in [10] as the subgeometries of free-simplicial geometries.

The modular flats of transversal pregeometries are characterized in [10] using (3.11), (3.16), and (5.7) of the present paper.

\section{Modular constructions.}

Definition 5.1. An injective pushout $P$ of $G$ and $H$ relative to $x$ is a colimit [21] for the diagram

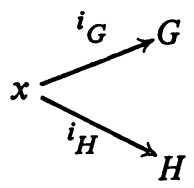

where the strong maps $i_{G}$ and $i_{H}$ are non-rank-decreasing monomorphisms. We may then identify $x$ with its images in $G$ and $H$ respectively which are subgeometries isomorphic to $x$ by (9.15) of [16]. This is a pushout for the category $G$ of geometries and strong maps.

Injective pushouts have been known to exist for $x$ equal to the empty geometry [16] in which case it is the coproduct (direct sum); and for $x$ equal 
to a point [6] in which case it is the parallel connection. Crapo asked for general criteria guaranteeing pushouts [15] and we will see in (5.3) that the modularity of $x$ in either $G$ or $H$ is such a guarantee for an injective pushout.

If $L$ is the rank two geometry (line) with the points $(G-x) \cup(H-x)$ $\cup x$ then the obvious maps from $G$ and $H$ into $L$ are strong, so that by the unique existence of the colimit map $P \rightarrow L$, we see that the points of an injective pushout when it exists can be identified with the point set $(G-x) \cup$ $(H-x) \cup x$ so that it is a geometry even in the category of pregeometries and strong maps. Further, let $\left\{\varnothing=x_{1}, \cdots, x_{k}=x\right\}$ be a saturated chain of closed sets in $x$; $\left\{\varnothing=g_{1}, \cdots, g_{i}=\bar{x}_{i}^{G}, \cdots, g_{k}=\bar{x}^{G}, g_{k+1}, \cdots, g_{n}=G\right\}$ be such a chain in $G$; and $\left\{\varnothing=h_{1}, \cdots, h_{i}=\bar{x}_{i}^{H}, \cdots, h_{k}=\bar{x}^{H}, h_{k+1}, \cdots\right.$, $\left.h_{m}=H\right\}$ a saturated chain in $H$. Then there can be no closed set $y$ of $P$ such that $y_{i} \subsetneq y \subsetneq y_{i+1}$ in the chain of sets $\left\{y_{1}, \cdots, y_{n+m-k}\right\}$ where $y_{i}=g_{i} \cup h_{i}$ for $i \in[1, k] ; y_{i}=g_{i} \cup h_{k}$ for $i \in[k, n]$; and $y_{i}=G \cup h_{i+k-n}$ for $i \in[n, n+m-k]$. Hence $r(P) \leqslant r(G)+r(H)-r(x)$. Let $G$ and $H$ be geometries with a common subgeometry $x$. The strong join $J$ of $G$ and $H$ relative to $x$ is the geometry on the points $(G-x) \cup(H-x) \cup x$ with closed sets all subsets $k$ such that $k \cap G$ is closed in $G$ and $k \cap H$ is closed in $H$, if the above closed sets form a geometric closure system. We remark that it is equivalent to consider all $k_{1} \cup k_{2} \cup k_{3}$ where $k_{1} \subseteq G-x, k_{2} \subseteq x$, and $k_{3} \subseteq H-x$ such that $k_{1} \cup k_{2}$ is closed in $G$ and $k_{2} \cup k_{3}$ is closed in $H$. Also these subsets include $J$ and all intersections so we need only check that they satisfy the exchange property.

Proposition 5.2. If a strong join $J$ exists for $G$ and $H$ relative to $x$, it is the injective pushout and its rank is equal to $r(G)+r(H)-r(x)$.

Proof. We have constructed the maximal family of closed sets for a geometry which completes the pushout diagram. Hence by the remarks in (5.1) it must be an injective pushout. Further, the closed sets include the saturated chain of (5.1) of length $r(G)+r(H)-r(x)$.

THEOREM 5.3. If $G$ and $H$ are geometries where $x$ is a modular flat of $G$ and a subgeometry of $H$ respectively, then the strong join of $G$ and $H$ exists, termed the generalized parallel connection, $P=P_{x}(G, H)$.

Proof. We show that the closed sets of $P_{x}(G, H)$ specified by a strong join form a geometric closure system. It is clearly a closure system by the remarks preceding (5.2).

We must show that in the lattice of closed sets, $L(P)$, for any closed set $k \in P$ and $p \notin k, k \vee p$ covers $k$. By our construction $k_{1}$ covers $k_{2}$ iff $k_{1} \cap G \geqslant k_{2} \cap G$ as closed sets of $G$, and $k_{1} \cap H \geqslant k_{2} \cap H$. The only pair 
of closed sets, one from the $G$ interval $\left[k_{1} \cap G, k_{2} \cap G\right]$ and the other from the $H$ interval $\left[k_{1} \cap H, k_{2} \cap H\right]$ which intersect $x$ in the same set are the two bottom elements and the two top elements respectively (note that either but not both intervals could be trivial).

To simplify the notation, for any subset $L$ of $P$, let $L_{G}, L_{H}$, and $L_{x}$ denote the subsets $L \cap G, L \cap H$, and $L \cap x$ respectively. Note that $L$ is closed iff $L_{G}$ and $L_{H}$ both are in the respective subgeometries (and hence so is $L_{x}$ since $x$ is closed in $G$ ). Also, $\mathrm{V}, \mathrm{V}_{G}, \mathrm{~V}_{H}$, and $\mathrm{V}_{x}$ denote the suprema operations in the subgeometries $P, G, H$, and $x$ respectively. We now derive an expression for $k \vee p$.

If $p \in G-x$ and $\left(k_{G} \vee_{G} p\right)_{x}=k_{x}$ then clearly $k^{\prime}=\left(k_{G} \vee p\right) \cup k_{H}$ contains $k \cup p$ and has intersection $k_{x}$ with both $G$ and $H$, while $\left[k_{G}, k_{G}^{\prime}\right]$ is a two point interval, and $\left[k_{H}, k_{H}^{\prime}\right]$ is a one point interval. Hence $k^{\prime}$ covers $k$ in the lattice of closed sets of $P$. A similar argument holds if $p \in H-x$ and $\left(k_{H} \vee_{H} p\right)_{x}=k_{x}$.

If $p \in G-x$ and $\left(k_{G} \vee_{G} p\right)_{x}>k_{x}$, let $q \in\left(k_{G} \vee_{G} p\right)-k_{x}$. Then $k_{G} \vee_{G} q=k_{G} \vee_{G} p$ so that $k \vee p$ contains both $k$ and $q$ and hence contains $k \vee q$ while $k \vee q$ contains $k \vee p$, so that $k \vee p=k \vee q$. A similar argument holds if $p \in H-x$ and $\left(k_{H} \vee_{H} p\right)_{x}>k_{x}$. Without loss of generality we may then assume that $p$ is in $x$. Let $y$ denote the flat of $x$ (and hence of $G)\left(k_{H} \vee_{H} p\right)_{x}$. Then we claim $k \vee p=k^{\prime}=\left(k_{G} \vee_{G} y\right) \cup\left(k_{H} \vee_{H} p\right)$. Surely $(k \vee p)_{H}$ must be closed in $H$ and contain $k_{H}$ and $p$ so that it must contain $k_{H} \vee_{H} p$. Hence $k \vee p$ contains $k_{H} \vee_{H} p$ and therefore contains $y$. But this means that $(k \vee p)_{G}$ must contain $k_{G}$ and $y$ and so must contain $k_{G} \vee_{G} y$. Hence we have $k \vee p \supseteq k^{\prime} \supseteq k \cup p$. But $k^{\prime}$ is a closed set of $P$ since by definition, $y=\left(k_{H} \vee_{H} p\right)_{x}$ and by modularity of $x$ in $G$ and (3.3.2),

$$
\left(y \vee_{G} k_{G}\right)_{x}=\left(y \vee_{G} k_{G}\right) \wedge_{G} x=y \vee_{G}\left(k_{G} \wedge_{G} x\right)=y \vee_{G} k_{x}=y \text {. }
$$

It remains to show that $k^{\prime}$ covers $k$. Assume $k^{\prime} \geqslant k^{\prime \prime}>k$. If $q \in\left(k^{\prime \prime}-k\right)$ $\cap H$, then as above $p \in k \vee q$ so $p \in k_{H} \vee_{H} q$ and by exchange in $H, k_{H} \vee_{H} q=$ $k_{H} \vee_{H} p$ so that $k^{\prime}=k^{\prime \prime}$. We may assume then that $q \in G-x$. But then

$$
q \in\left(k_{G} \vee_{G} y\right)-k_{G} \subseteq \overline{k_{G} \cup x}-k_{G}
$$

so that by the modular exchange axiom (3.17) there exists $q^{\prime}$ such that

$$
q^{\prime} \in\left({\overline{k_{G} \cup q^{G}}}^{G} k_{G}\right)_{x} \subseteq k_{x}^{\prime \prime}-k_{x} \subseteq\left(k^{\prime \prime}-k\right) \cap H
$$

and we are done as above.

EXAMPLES 5.4. Although every generalized parallel connection is a strong join and every strong join is a pushout for our category $G$, the following counter- 
examples show that converses to (5.2) and (5.3) are not in general true.

(1)
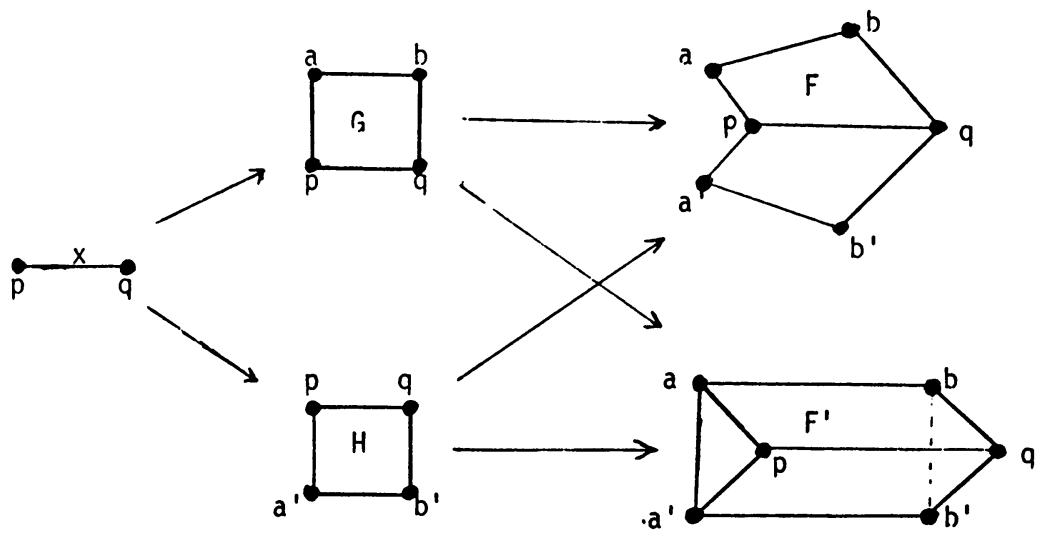

The above example shows that injective pushouts need not exist since the pushout of $G$ and $H$ relative to $x$ must be one the point set $\left\{p, q ; a, b ; a^{\prime}, b^{\prime}\right\}$ and have rank at most $r(G)+r(H)-r(x)=4$. However, referring to the above diagram, if there were a strong map from $P$ to $F,\left\{a, b, a^{\prime}\right\}$ would be closed in $P$; while if there were a strong map from $P$ to $F^{\prime}, \varnothing,\{a\},\{a b\},\left\{a, b, a^{\prime}, b^{\prime}\right\}$, and $\left\{a, b, a^{\prime}, b^{\prime}, p, q\right\}$ would all be closed so that $P$ would have rank at least five. Hence $P$ cannot exist.

(2)

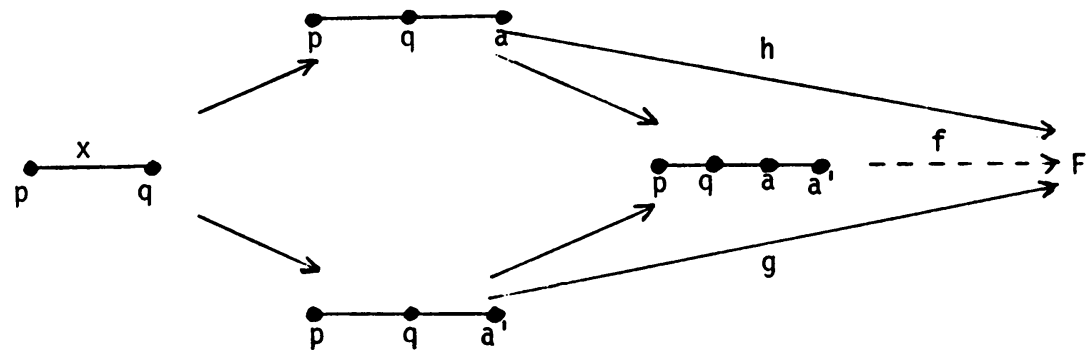

The above example shows that injective pushouts can exist which are not strong joins. In $P,\left\{a, a^{\prime}\right\}$ is not closed although $\left\{a, a^{\prime}\right\} \cap G=a$ is closed in $G$ while $\left\{a, a^{\prime}\right\} \cap H=a^{\prime}$ is closed in $H$. However, if $F$ is any geometry with strong maps $g$ and $h$ into it, then letting

$$
\begin{array}{ll}
f(p)=g(p)=h(p), & f(a)=g(a), \\
f(q)=g(q)=h(q), & f\left(a^{\prime}\right)=h\left(a^{\prime}\right),
\end{array}
$$

$f$ is a strong map since $a \in \overline{p q}$ because $g$ is strong, while $a^{\prime} \in \overline{p q}$ because $h$ is strong so that $\left\{p, q, a, a^{\prime}\right\}$ would have rank two and no proper subset of $\left\{p, q, a, a^{\prime}\right\}$ (except $\varnothing$ and the points) could be closed. 


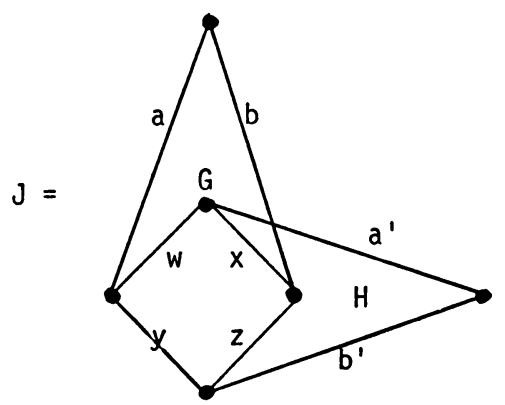

The above graphical example shows that while one may check that $J$ is the strong join of the graphs $G=\{a, b, w, x, y, z\}$ and $H=\left\{a^{\prime}, b^{\prime}, w, x, y, z\right\}$ relative to the common closed subgraph $\{w, x, y, z\}$; this subgraph is modular in neither $G$ nor $H$ by (4.3.2).

(4) In light of example (5.4.1) it seems an interesting problem to determine if pushouts exist for the category $P$ of geometries (or pregeometries) and injective (weak) maps (i.e. injections on points such that preimages of independent sets are independent). This problem is equivalent to determining if any colimit diagram of (5.1) can be completed by a (pre)geometry $F$ on the set of points $(G-x) \cup$ $x \cup(H-x)$ such that if any other pregeometry $F^{\prime}$ on the same set of points completes the diagram, then every independent set of $F^{\prime}$ is independent in $F$.

In (5.4.1), $F^{\prime}$ (and in fact any geometry which completes the pushout diagram) is a weak map image of $F$. However, the following example shows that in general pushouts do not exist even in the category $P$.

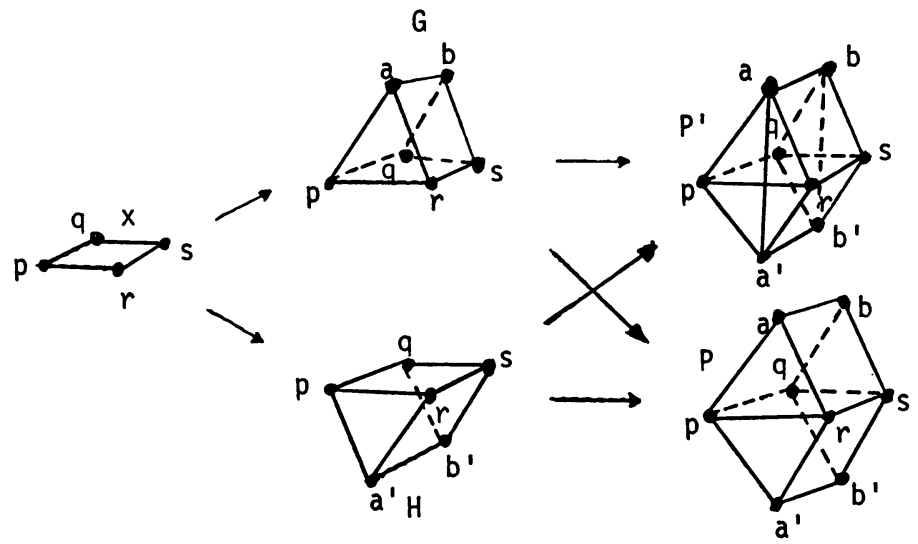

Both $G$ and $H$ are isomorphic to $F^{\prime}$ of (5.4.1). $P$ is the rank four geometry generated by the five connected planes $\{p, q, r, s\},\{p, q, a, b\},\left\{p, q, a^{\prime}, b^{\prime}\right\}$, $\{r, s, a, b\}$, and $\left\{r, s, a^{\prime}, b^{\prime}\right\}$. No rank 5 geometry has this planar structure.

On the other hand, $P^{\prime}$ is a rank 5 geometry which when truncated to rank 
4 (as pictured) is the same geometry as $P$ with the additional dependence $\left\{a, b, a^{\prime}, b^{\prime}\right\}$. Both $P$ and $P^{\prime}$ have $G$ and $H$ as subgeometries and no weak map exists between them. But clearly a weak pushout if it existed would necessarily have the planar structure of $P$ and the rank of $P^{\prime}$, hence none exists.

Proposition 5.5. 1. If $k$ is closed in $P=P_{x}(G, H)$ then $r(k)=r_{G}\left(k_{G}\right)+$ $r_{H}\left(k_{H}\right)-r_{G}\left(k_{x}\right)$. In particular, $r(P)=r(G)+r(H)-r(x)$.

2. If $g \subseteq G$ and $h \subseteq H$, then the closure of $g \cup h$ in $P, \overline{g \cup h}$, equals

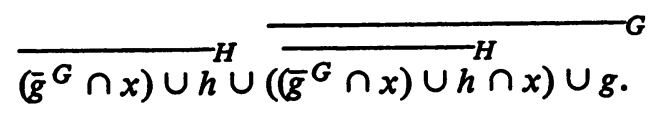

3. $r_{P}(g \cup h)=r_{H}\left(h \cup\left(\bar{g}^{G} \cap x\right)\right)+r_{G}(g \cup x)-r_{G}(x)$.

4. $g \cup h$ is independent in $P$ if and only if $g$ is independent in $G$ and $h-x$ is independent of $g \cap x$ in $H$.

Proof. (1) follows from the remarks in (5.1) and (5.2); and from the fact that generalized parallel connections are strong joins.

To prove (2) we note that $\overline{g \cup h}$ must contain $\bar{g}^{G}$ and hence $\bar{g}^{G} \cap x \subseteq x$ $\subseteq H$. Therefore, since it is closed in $H$ it must contain

$$
h^{\prime}=\overline{\left(\bar{g}^{G} \cap x\right) \cup h} \text {. }
$$

Hence it contains $h^{\prime} \cap x \subseteq x \subseteq G$ and hence must contain

$$
\left.g^{\prime}={\overline{\left(\overline{\left(\bar{g}^{G} \cap x\right) \cup h}\right.}}^{H} \cap x\right) \cup g .
$$

But $g^{\prime} \cap x=\left(\left(h^{\prime} \cap x\right) \vee \bar{g}\right) \wedge x=\left(h^{\prime} \cap x\right) \vee(\bar{g} \wedge x)=h^{\prime} \cap x$ so that the subset specified in (2) is closed.

(3) Let

$$
\left.u=\overline{(\bar{g}}^{G} \cap x\right) \cup h{ }^{H} \cap x
$$

which is a closed subset of the subgeometry $x$ and hence a flat of $g$ containing $\bar{g} \wedge x$. Therefore

$$
r(g \cup h)=r(\overline{g \cup h})=r_{H}\left(h \cup\left(\bar{g}^{G} \cap x\right)\right)+r_{G}(u \cup g)-r_{G}(u) .
$$

But in $G$,

$$
\begin{aligned}
r(u \cup g)-r(u) & =r(u \vee \bar{g})-r(u) \\
& =[r((u \vee \bar{g}) \wedge x)+r(\bar{g} \vee u \vee x)-r(x)]-r(u)
\end{aligned}
$$

(by the modularity of $x$ in $G$ )

$$
=r(u)+r(g \cup x)-r(x)-r(u) \text {. }
$$


Finally, in (4), $h-x$ is independent of $\bar{g} \cap x$ in $H$ if and only if

$$
r_{H}((\bar{g} \cap x) \cup h)=r_{H}(\bar{g} \cap x)+|h-x| .
$$

Hence $r(g \cup h)=|g \cup h|=|g|+|h-x|$ if and only if

$$
\begin{aligned}
r_{H}(h \cup(\bar{g} \cap x)) & +\left(r_{G}(g)-r_{G}(\bar{g} \cap x)\right) \\
& =(r(\bar{g} \cap x)+|h-x|)+(|g|-r(\bar{g} \cap x))
\end{aligned}
$$

if and only if equality holds between each respective summand.

DEFINITION 5.6. A single element extension as studied by Crapo [16] of a geometry $G$ by a point $p$ is a geometry $G^{\prime}$ such that $r\left(G^{\prime}\right)=r(G)$, and $G=G^{\prime}-$ $p$. More generally, an extension of a geometry $G$ is a sequence of single element extensions (i.e. a geometry which contains $G$ as a spanning subgeometry).

Proposition 5.7. If $x$ is a modular flat of a geometry $G$ and $x^{\prime}$ is an extension of $x$ then $G^{\prime}=P_{x}\left(G, x^{\prime}\right)$ is the unique extension of $G$ such that $x^{\prime}$ is a flat of $G^{\prime}$ and $G^{\prime}-\left(x^{\prime}-x\right)=G$. We call this special case the modular extension of $G$ by $x^{\prime}$.

Proof. $G^{\prime}=P_{x}\left(G, x^{\prime}\right)$ is an extension of $G$ by $x^{\prime}-x$ since it contains $x^{\prime}$ as a flat, and $G$ as a subgeometry which spans since $r\left(G^{\prime}\right)=r(G)+r\left(x^{\prime}\right)-r(x)=$ $r(G)$. But any other extension $G^{\prime \prime}$ would complete the pushout diagram and hence there would be a bijective strong map from $G^{\prime}$ to $G^{\prime \prime}$. But $r(G)=r\left(G^{\prime \prime}\right)=r\left(G^{\prime}\right)$ and hence $G^{\prime}$ would be isomorphic to $G^{\prime \prime}$ by $(9.15)$ of [16].

Further, $x^{\prime}$ is modular in $G^{\prime}$ since if $u$ were a flat disjoint from $x^{\prime}$ it would be closed and disjoint from $x$ in $G$ so that

$$
\begin{aligned}
r_{G^{\prime}}\left(x^{\prime}\right)+r_{G^{\prime}}(u) & =r_{G}(x)+r_{G}(u)=r_{G}(x \vee u)=r_{G}(x \cup u) \\
& =r_{G^{\prime}}(x \cup u)=r_{G^{\prime}}\left(x^{\prime} \cup u\right)=r_{G^{\prime}}\left(x^{\prime} \vee u\right) .
\end{aligned}
$$

REMARKs 5.8. By (5.7) we note that any generalized series-parallel connection factors uniquely first as a modular extension and then as a generalized parallel connection relative to a subset which is modular in $G$ and a flat of $H$ :

$$
P_{x}(G, H)=P_{\bar{x}^{H}}\left(P_{x}\left(G, \bar{x}^{H}\right), H\right) .
$$

We call the latter connection (when the subgeometry is closed in $H$ ) a closed connection. For such closed connections, $G, x$, and $H$ are all flats of $P$.

We can now give a lattice characterization of closed connections. 
Proposition 5.9. A geometry $P$ is a closed connection of flats $G$ and $H$ relative to a flat $x$ if and only if $[x, P]=[x, G] \oplus[x, H]$ and $x$ is a modular flat of the subgeometry $G$ (equivalently, $H$ is a modular flat of $P$ ).

More generally, a geometry $P$ is equal to $P_{x}(G, H)$ iff $x$ is a modular flat of $G$ and $\left[\bar{x}^{P}, P\right]=[x, G] \oplus\left[\bar{x}^{H}, H\right]$.

Proof. The fact that $x$ is a modular flat in a subgeometry $G$ if and only if $H$ is modular was proved in (3.19). Clearly the conditions are necessary since $[x, P]=[x, G] \oplus[x, H]$ if and only if the points of $P$ are partitioned into those lying below $G-x, x$, and $H-x$ respectively and a set $A$ containing $x$ is closed iff $A \cap G$ is closed in $G$ and $A \cap H$ is closed in $H$. Such always happens in a closed connection while by definition $x$ is modular in $G$.

Conversely, assume the conditions of the proposition hold. We are given that $x$ is modular in $G$. Also, for all flats $y$ of $P, y \cap G=y \wedge G$ is a flat of $P$ as is $y \cap H=y \wedge H$ while $(y \wedge G) \wedge x=y \wedge x=(y \wedge H) \wedge x$. Hence we need only to show that if $y_{G}$ is a flat of the subgeometry $G$ and $y_{H} \leqslant H$ such that $y_{G} \wedge x=y_{H} \wedge x=y_{x}$ then $y_{G} \vee y_{H}=y_{G} \cup y_{H}$. Since $G \cup H=$ $P$ we will be done if we show

$$
\left(y_{G} \vee y_{H}\right) \cap G=\left(y_{G} \vee y_{H}\right) \wedge G=y_{G} \quad \text { and } \quad\left(y_{G} \vee y_{H}\right) \wedge H=y_{H} .
$$

But since $H$ is modular,

$$
\left(y_{G} \vee y_{H}\right) \wedge H=y_{H} \vee\left(H \wedge y_{G}\right)=y_{H} \vee\left(x \wedge y_{G}\right)=y_{H} \vee y_{x}=y_{H} .
$$

Further, $\left(y_{G} \vee y_{H}\right) \wedge G$ lies in the interval $\left[y_{G}, G\right]$. By the modularity of $x$ in $G, \Lambda_{x}$ is an isomorphism from $\left[y_{G}, G\right]$ to $\left[y_{x}, x\right]$. But

$$
\left(\left(y_{G} \vee y_{H}\right) \wedge G\right) \wedge x=\left(y_{G} \vee y_{H}\right) \wedge x \leqslant\left(y_{G} \vee y_{H}\right) \wedge H=y_{H}
$$

so that $\left(y_{G} \vee y_{H}\right) \wedge x \leqslant y_{H} \wedge x=y_{x}$. Hence $\left(y_{G} \vee y_{H}\right) \wedge G$ meets $x$ in $y_{x}$ and has the same image under the isomorphism $\Lambda x$ as $y_{G}$. Hence $\left(y_{G} \vee y_{H}\right) \wedge G=y_{G}$ and so $y_{G} \vee y_{H}=y_{G} \cup y_{H}$.

The second part of the proposition then follows from the first part and (5.8).

Proposition 5.10. In the generalized parallel connection $P_{x}(G, H)$, the following hold:

1. $H$ is a modular flat.

2. $G$ and $x$ are both subgeometries. 
3. $G$ is a flat if and only if $x$ is a flat if and only if the connection is closed.

4. $G$ is a modular flat if and only if $x$ is a modular flat if and only if $x$ is a modular flat of $H$.

5. If $G$ is connected, then $P$ is connected if and only if $H$ is connected (and $x \neq \varnothing$ ).

6. If $x$ is also a modular flat of $H$, then $P_{x}(H, G)=P_{x}(G, H)$.

7. If $x$ is also a modular flat of $H$ and a subgeometry of $F$, then $P_{x}\left(P_{x}(G, H), F\right)=P_{x}\left(G, P_{x}(H, F)\right)$.

8. If $G$ is a modular flat of $G^{\prime}$, then $P_{x}\left(G^{\prime}, H\right)=P_{G}\left(G^{\prime}, P_{x}(G, H)\right)$.

Proof. The first statement follows from (5.8) where it was shown that $P_{x}(G, H)=P_{\bar{x}}\left(P_{x}\left(G, \bar{x}^{H}\right), H\right)$. But the latter term is a closed connection with $\bar{x}^{H}$ modular in $P_{x}\left(G, \bar{x}^{H}\right)$ so that we may apply (3.19) or (5.9).

(2) The closed sets of $P$ when intersected with $G$ are closed and every closed set $k$ of $G$ can be realized as such an intersection of $G$ with a closed set of $P$, e.g. $\left(k \cup(\overline{k \cap x})^{H}\right) \cap G=k$. Further $x$ is a subgeometry of $G$ and hence of $P$.

(3) Each part is equivalent to the modular extension in (5.8) being trivial.

(4) By (3) we may assume the connection is closed in which case we apply (1), (3.5), (3.6), and (3.19).

(5) If $G$ is connected, then $x$ must be connected by (3.16). Hence, if $H$ is separable, $\bar{x}^{H}$ must lie in one of its components and any other component will remain a separator of $P$. Conversely, assume $G$ and $H$ are both connected and $x \neq \varnothing$ (if $x=\varnothing, P_{x}(G, H)$ is the direct sum and hence separable). Then, since $G$ and $H$ are both connected subgeometries, any separator which intersects either must contain it. But $G \cap H \neq \varnothing$, so no separator can intersect $G \cup H$ nontrivially.

(6) Trivial from the symmetry in the properties of (5.2).

(7) Both sides are well-defined since $x$ is a modular flat of $P_{x}(G, H)$ by (4). Also the point sets on both sides are given by $G \cup H \cup F$ and the closed sets by sets which are closed in the respective geometries when intersected with $G, H$ and $F$.

(8) The left-hand side is well-defined since $x$ is a modular flat of $G^{\prime}$ by (3.5). Further, both sides are geometries on the points $G^{\prime} \cup H$ while a set is closed on the left iff its intersections with $G^{\prime}$ and $H$ are both closed. But if its intersection with $G^{\prime}$ is closed, so is its intersection with $G$ (since $G$ is a flat of $G^{\prime}$ ) so that a set is closed on the left iff its intersections with $G^{\prime}, G$, and $H$ are all closed and hence iff its intersections with $G^{\prime}$ and $P_{x}(G, H)$ are both closed iff it is closed in the right-hand side connection. 
Proposition 5.11. For all $p \in G-x, q \in H-x$, and $s \in x$, the following commutativity properties hold:

1. $P_{x}(G, H)-p=P_{x}(G-p, H)$.

2. $P_{x}(G, H)-q=P_{x}(G, H-q)$.

3. $\left[p, P_{x}(G, H)\right] \simeq P_{[p, x \vee p]}([p, G], H)$.

4. If $x$ is a flat in $H,\left[q, P_{x}(G, H)\right] \simeq P_{x}(G,[q, H])$.

5. $\left[s, P_{x}(G, H)\right] \simeq P_{[s, x]}([s, G],[s, H])$.

Proof. Both sides of (1) are defined on the point set $(G-x-p) \cup x$ $\cup H-x ; x$ is modular in $G-p$ by (3.8) and sets are closed in either side iff their intersections with $G-p$ and $H$ are closed in the respective geometries.

Similarly, both sides of (2) are defined on the set of points $G-x \cup x$ $\cup H-p-x$ and have closed sets exactly those whose intersections with $G$ and $H-p$ are closed respectively.

The atoms on the left side of (3) correspond to lines of $P_{x}(G, H)$ covering $p$. Among these atoms there is an isomorphic copy of $x$ by (3.3.8); an isomorphic copy of $H$ by (3.3.8) and (5.10.1); and the rest of the atoms correspond to lines contained in $G$ but not in $x \vee p . \quad[p, x \vee p]$ is modular in $[p, G]$ by (3.3.8) and flats in both cases correspond to flats which contain $p$ and intersect both $G$ and $H$ in flats.

To prove (4) we note that in $P_{x}(G,[q, H])$ the subgeometry $x$ corresponds to the subgeometry arising from the image of $x$ under the strong map $V_{q}$ which one checks is isomorphic to $x$ in $H$ (and hence in $P_{x}(G ; H)$ ). The isomorphism between the two sides then follows noting that if all intersections behave properly, then they also do for flats containing $q$.

(5) $[s, x]$ is modular in $[s, G]$ by (3.9). The isomorphism can then best be seen by considering the interval $[s, P]$ relative to (5.9).

REMARKS 5.12 (The comap join of $H$. Crapo). The generalized parallel connection is an instance of a more general construction of Crapo: the comap join of two geometries [13].

In Crapo's work a function from a geometric lattice $L(G)$ into a geometric lattice $L(H)$ is a comap if it is chain contracting (i.e. covering pairs are preserved under one-one images) and it preserves meets of modular pairs. Examples of comaps include composites of comaps; the natural imbedding of a subgeometry $G^{\prime} \subseteq G$ (where $L\left(G^{\prime}\right)$ is realized as the join subsemilattice of $L(G)$ ); and the canonical retract of a geometry $G$ onto a modular flat $x$, $c: L(G) \rightarrow[0, x]$ where $c(y)=x \wedge y$. In fact it is the modularity of $x$ which makes the map $c$ chain contracting (cf. 3.3.4 above).

If $c_{1}$ and $c_{2}$ are comaps which both preserve 0 , the comap join of two geometries $G$ and $H$ across comaps $c_{1}: L(G) \rightarrow L(F)$ and $c_{2}: L(H) \rightarrow$ 
$L(F)$ can be seen to consist of the geometry on the disjoint union $\{G\} \cup\{H\}$ of the points (atoms) of $G$ and $H$ respectively whose rank function is given for all $g \subseteq G, h \subseteq H$ by

$$
\begin{aligned}
r(g \cup h) & =r_{G}(g)-r_{F}\left(c_{1}\left(\bar{g}^{G}\right)\right)+r_{H}(h) \\
& -r_{F}\left(c_{2}\left(\bar{h}^{H}\right)\right)+r_{F}\left(c_{1}\left(\bar{g}^{G}\right) \cup c_{2}\left(\bar{h}^{H}\right)\right) .
\end{aligned}
$$

If $x$ is a modular flat of $G$ and a subgeometry of $H$ we have the comaps $c_{2} \circ c_{1}$ and $c_{3}$ where $c_{1}: L(G) \rightarrow[0, x]$ is the canonical retract, $c_{2}: L(x) \rightarrow L(H)$ is the natural imbedding of the subgeometry $x$ into $H$, and $c_{3}: L(H-x) \rightarrow L(H)$ is the natural imbedding of the subgeometry $H-x$ into $H$. Then since the rank function for subsets of $H-x$ is identical in $L(H)$ and $L(H-x)$, and the rank function for subsets of $x$ is identical in $L(x), L(H)$, and $L(G)$; if $g \subseteq G, h \subseteq H$, and $g \cap x=h \cap x$, (*) becomes:

$(* *)$

$$
\begin{aligned}
r(g \cup h)= & r(g \cup(h-(h \cap x)))=r_{G}(g)-r_{G}\left(\bar{g}^{G} \cap x\right) \\
& \left.+r_{H}\left(\left(\bar{g}^{G} \cap x\right) \cup \overline{\left(h-(h \cap x)^{H}\right.}\right)\right) \\
= & r_{G}(g \cup x)-r_{G}(x)+r_{H}\left(\left(\bar{g}^{G} \cap x\right) \cup(h-(h \cap x))\right) \\
= & r_{G}(g \cup x)-r_{G}(x)+r_{H}\left(\left(\bar{g}^{G} \cap x\right) \cup h\right),
\end{aligned}
$$

using the facts that $h \cap x \subseteq \bar{g}^{G} \cap x, r_{H}\left(h^{\prime} \cup h\right)=r_{H}\left(h^{\prime} \cup \bar{h}\right)=r_{H}\left(\bar{h}^{\prime} \vee \bar{h}\right)$, and the fact that $x$ is modular in $G$.

But (**) is the same as (5.5.3) for the rank function of the generalized parallel connection $P_{x}(G, H)$.

Many of the other properties proved for the generalized parallel connection hold more generally in the context of comap joins. For example, (5.10.6), (5.10.7), and (5.10.8) have join-theoretic analogs while [14] includes a computation of the Tutte polynomial [7] across a join of graphs which reduces to (7.13) below in our case.

In light of the categorical properties of the generalized parallel connection it would seem fruitful to explore the join operation, and possibly resolve the general question of when injective pushouts exist for weak or strong maps.

Another construction in which modularity plays a simplifying role is the Brown truncation.

Definition 5.13. For a flat $x$ of a geometry $G, T_{x}(G)$, the Brown truncation of $G$ relative to $x$ is the geometry whose geometric lattice is given by deleting from $L(G)$ the "collar" of the principal order filter generated by $x$ (i.e. those flats $y$ such that $y$ is covered by $x \vee y$ ). This operator was defined in [5] and is explored in [9]. The rank function, $r_{T}$, for the remaining closed sets in $T_{x}(G)$ is given by $r_{T}(y)=r(y)-1$ if $y \geqslant x$ and $r_{T}(y)=r(y)$ if $y \geqslant x$. 
Further, the bases of $T_{x}(G)$ are those subsets $B-p$ where $B$ is a basis of $G$ and $p \in B \cap x$. The flat $x$ in $T_{x}(G)$ is isomorphic to the upper rank one truncation $T(x)$ of $x$ (as a flat of $G$ ). We let $T^{k}(x)$ denote the upper rank $k$ truncation of $x$.

The complete Brown truncation of $G$ relative to a flat $x, \bar{T}_{x}(G)$, is equal to

$$
T_{T^{n-2}(x)}\left(T_{T^{n-3}(x)}\left(\cdots\left(T_{x}(G)\right) \cdots\right)\right)
$$

where $r(x)=n$ and hence $x$ is truncated $n-1$ times (and becomes an atc.n). Geometrically, $\bar{T}_{x}(G)$ corresponds to the extension of $G$ by a flat put in general position as a hyperplane of the subgeometry $x$ followed by the contraction of that hyperplane.

Proposition 5.14. Assume $r(x)>1$ and $x$ is a modular flat of $G$. Then the following hold:

1. $x$ is a modular flat of $T_{x}(G)$.

2. For all $p \notin x, T_{x}(G)-p=T_{x}(G-p)$, and $\left[p, T_{x}(G)\right] \simeq$ $T_{[p, x \vee p]}([p, G])$. Also, $p$ is an isthmus of $G$ iff $p$ is an isthmus of $T_{x}(G)$.

3. $\bar{T}_{x}(G)$ as a geometric lattice is isomorphic to the lattice formed by the flats $\{y \mid y \geqslant x\} \cup\left\{y^{\prime} \mid y^{\prime} \wedge x=0\right\}$ with the induced order from $G$. Hence $G$ is isomorphic to a geometry whose flats are the subsets $\{y\} \cup\left\{y^{\prime}-x \cup q\right\}$ for all $y \cap x=\varnothing$ and $y^{\prime} \supseteq x$.

PRoof. (1) In $T_{x}(G)$, if $y \geqslant x$, then $r_{T}(x)+r_{T}(y)=(r(x)-1)+$ $(r(y)-1)=(r(x \vee y)-1)+(r(x \wedge y)-1)=r_{T}(x \vee y)+r_{T}(x \wedge y)$ (since $x \vee y$ and $x \wedge y$ are both greater than or equal to $x$ ). On the other hand, if $y \neq x$, then $y \vee x$ does not cover $y$ (since $x$ is modular and has rank greater than one) but $y \vee x>x$, hence $y \vee x$ is a flat of $T_{x}(G)$. Also $(x \wedge y) \vee x$ equals $x$ which cannot cover $x \wedge y$ by the modularity of $x$, so that $x \wedge y$ is a flat of $T_{x}(G)$ but is not greater than or equal to $x$. Hence

$$
\begin{aligned}
r_{T}(x)+r_{T}(y) & =(r(x)-1)+r(y) \\
& =(r(x \vee y)-1)+r(x \wedge y)=r_{T}(x \wedge y)+r_{T}(x \wedge y)
\end{aligned}
$$

and $x$ is modular in $T_{x}(G)$.

(2) We note that since $r(x)>1$, if $p \notin x, x \vee p$ does not cover $p$ and hence $p$ is a flat of $T_{x}(G)$. Then for all $p \notin x$, if $p$ is not an isthmus of $G$ there is a basis $B$ which does not contain $p$. If $B$ intersects $x$, then $B-q(q \in B \cap x)$ is a basis of $T_{x}(G)$ which does not contain $p$ and $p$ is 
not an isthmus of $T_{x}(G)$. If $B \cap x=\varnothing$, and if $B^{\prime}$ is any basis which intersects $x$ but contains $p$, we may use the basis exchange axiom to find a new basis $\left(B^{\prime}-p\right) \cup q(q \in B)$ which intersects $x$ and does not contain $p$. Hence $p$ is not an isthmus of $G$. Conversely, if all bases of $G$ contain $p$, so do all bases of $T_{x}(G)$ and $p$ is an isthmus of $T_{x}(G)$.

$T_{x}(G)-p$ and $T_{x}(G-p)$ are both geometries on the set $\{G\}-p$ whose bases are those subsets $B-q$ of $G$ which do not contain $p$ and with $q \in x$ $\cap B$. Further $\left[p, T_{x}(G)\right]$ and $T_{[p, x \vee p]}([p, G])$ are the geometries associated with the pregeometry whose bases are of the form $B-q$ where $q \in B \cap x$ and $p \in B$.

(3) If $r(x)=n$, then the flats of $T_{x}(G)$ are those of $G$ except those $y$ such that $r(x \vee y)=r(y)+1$. But if $x$ is modular these are exactly those flats such that $r(x \wedge y)=r(x)-1$. By (1) above, $x$ is modular in this truncation; and when we truncate again, we remove those flats such that $r_{T}(x \wedge y)=$ $r_{T}(x)-1$, i.e. such that $r(x \wedge y)=r(x)-2$. Similarly, after the $(n-1)$ st truncation we have removed $y$ if and only if $r(x \wedge y)=r(x)-k$ where $k \in$ $[1, n-1]$, i.e. $1 \leqslant r(x \wedge y) \leqslant n-1$. But these are precisely all the flats which intersect $x$ but do not contain $x$.

6. Extensions and connections for classes of geometries. In this section we explore an extension property consistent with a given representation of a geometry (as a binary or ternary chain group, unimodular chain group, or graph). We then show how such an extension property guarantees that a generalized parallel connection of two geometries in a particular class can be constructed within that class.

DEFINITION 6.1. For a class $K$, a subgeometry $x$ of a geometry $G$ in $K$ has the extension property if whenever $x^{\prime}$ is a representation for $x$ there is a representation $G^{\prime}$ for $G$ such that $x^{\prime}$ is the canonically induced representation of $x$ from $G^{\prime}$.

If every pair $(G, x)$ where $x$ is a subgeometry of $G$ in $K$ has the extension property, we say $K$ has the general extension property, while if every pair $(G, x)$ where $x$ is a modular flat of $G$ in $K$ has the extension property, we say $K$ has the modular extension property.

Using the results of $\S 4$ we will assume in the following unless otherwise stated that all graphical representations are (path) connected and all matrix representations of $G$ have $r(G)$ rows.

The following examples show that chain groups and graphs do not have the general extension property while transversal geometries do not even have the modular extension property.

EXAMPLES 6.2. 1. The subgeometry $\{a, b, c, d, e, f\}$ of the geometry 


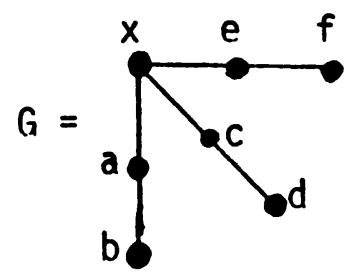

represented as a chain group over the integers (or rationals) by

$$
M=\left[\begin{array}{lllllll}
a & b & c & d & e & f & x \\
1 & 0 & 0 & 1 & 2 & 1 & 1 \\
0 & 1 & 0 & 1 & 3 & 2 & 1 \\
0 & 0 & 1 & 2 & 3 & 3 & 0
\end{array}\right]
$$

does not have the extension property since another representation for this subgeometry would be

$$
M^{\prime}=\left[\begin{array}{llllll}
a & b & c & d & e & f \\
1 & 0 & 0 & 1 & 2 & 3 \\
0 & 1 & 0 & 1 & 3 & 2 \\
0 & 0 & 1 & 2 & 3 & 3
\end{array}\right]
$$

while if this representation could be extended to a representation for $G$ by adjoining the column vector $\left(x_{1}, x_{2}, x_{3}\right)$ the line $a b x$ would guarantee $x_{3}=$ 0 ; the line $c d x$ would guarantee $x_{1}=x_{2}$ while the vector $(a, a, 0)$ cannot lie on a line with ef. We may in fact extend the above arguments to the matrices

$$
M^{\prime \prime}=\left[\begin{array}{llllllll}
1 & 0 & 0 & 1 & 2 & 1 & 0 & 1 \\
0 & 1 & 0 & 1 & 3 & 2 & 0 & 1 \\
0 & 0 & 1 & 2 & 3 & 3 & 0 & 0 \\
0 & 0 & 0 & 0 & 0 & 0 & 1 & 1
\end{array}\right]
$$

and $M^{\prime}$ which now represents a flat of the geometry represented by $M^{\prime \prime}$.

2. The question, however, of whether modular flats have the extension property $(6.8,6.9)$ is open and should prove an interesting research problem.

Since graphical representations of geometries whose graph is three-connected are unique, the flat $\{a, b\}$ in the (planar) graph 


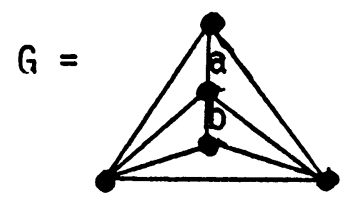

although representable graphically by two disconnected edges, must be adjacent in any graphical representation of $G$.

3. For transversal geometries, one readily checks that the (modular) point $a$ in the geometry represented by the relation

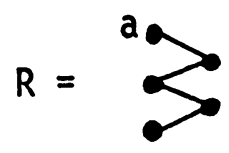

cannot have a representation

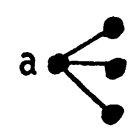

extended to a representation for $R$ so that the modular extension property does not hold for transversal geometries.

However, two important classes of geometries have the extension property while another has the modular extension property.

DEFINITION 6.3. A chain group class $K$ has the unique representation property if all $G \in K$ and matrix representations $M_{1}$ and $M_{2}$ (each with $r(G)$ rows) of $G$, then $M_{1}$ can be transformed into $M_{2}$ by a series of operations consisting of elementary row operations and multiplying columns by nonzero scalars. This is clearly an equivalence relation on matrices, denoted $M_{1} \sim M_{2}$.

Proposition 6.4. A chain group class $K$ with the unique representation property has the extension property.

Proof. Let $A$ be a subgeometry of $G \in K$. Assume a matrix $M$ which represents $G$ has columns $C$ which represent $A$. Then if $M^{\prime}$ is another matrix which represents $A$, filling out $M^{\prime}$ if necessary by adding zero rows we have $M^{\prime} \sim C$ by the unique representation property and we may perform these same operations in $M$ so that we obtain a new matrix representing $G$ such that a subset of its columns has the representation $M^{\prime}$.

PROPOSITION 6.5. The class $B$ of binary geometries has the unique representation property and hence the extension property when represented over any field. 
Proof. This is Theorem 3.7 of [11].

THEOREM 6.6. The classes of unimodular geometries, binary chain groups, and ternary chain groups have the unique representation property and hence the extension property.

Proof. Geometries in all three classes were shown to have unique representations in Theorem 3.2 of [11].

Proposition 6.7. In a chain group, points and direct sum factors have the extension property.

Proof. In any matrix, points correspond to nonzero vectors and suitable row operations can make any such vector into, for example, $(1,0,0, \cdots, 0)$. Further, if $G=x \oplus y$ and $M_{x}$ is any matrix representation for $x$ while $M_{y}$ is any representation for $y$, then

$$
M=\left(\begin{array}{ll}
M_{x} & 0 \\
0 & M_{y}
\end{array}\right)
$$

is a representation for $G$.

CONJECTURE 6.8. Chain groups have the modular extension property.

REMARKs 6.9. To lend some insight into (6.8) we note that it holds for those modular flats which are points or direct sum factors by (6.7) and for binary and ternary chain groups by (6.6).

More generally, if a geometry $G$ with a modular flat $x$ is representable by a matrix $M$, we may assume by employing row operations if necessary as in (4.1.2) that

$$
M=\left(\begin{array}{ccc}
M_{x} & 0 & M_{1} \\
0 & I & M_{2}
\end{array}\right)
$$

where $M_{x}$ represents the modular flat and (by suitable scalar multiplication of columns) each column $c_{j}$ of $M_{1}$ is zero or identical to a column $c_{i}$ of $M_{x}$. Then, if $M_{x}^{\prime}$ is another representation for $x$, the conjecture states that we may construct a representation of $G$ of the form

$$
M^{\prime}=\left(\begin{array}{lll}
M_{x}^{\prime} & 0 & M_{1}^{\prime} \\
0 & I & M_{2}^{\prime}
\end{array}\right)
$$


where the columns $c_{j}^{\prime}$ of $M_{1}^{\prime}$ will necessarily be zero if the corresponding column of $M_{1}$ was zero and will be identical to the column $c_{i}^{\prime}$ of $M_{x}^{\prime}$ if $c_{j} \in$ $M_{1}$ was identical to $c_{i} \in M_{x}$. Hence, the conjecture can be restated as saying that a suitable $M_{2}^{\prime}$ can be found.

Another reason to be hopeful that chain groups enjoy the modular extension property is the following analogous theorem for graphs.

THEOREM 6.10. Graphs have the modular extension property.

Proof. Let $F$ be the subgraph representing a modular flat of a graph $G$ and let $F^{\prime}$ be another graphical presentation of $F$. Then, by [25], $F$ may be transformed into $F^{\prime}$ by separating and reconnecting $F$ at cutpoints (*) or by separating $F$ at a two element cutset and reconnecting at the same two vertices after reversing the orientation of one of the pieces (**). Under these conditions $F$ and $F^{\prime}$ were termed two-isomorphic by Whitney.

But since cutpoints induce a direct sum decomposition of $F$, every cutpoint of $F$ is a cutpoint of $G$ by (3.16) so that any (*) operation of $F$ may be performed in $G$ to get a new graph $G^{\prime}$ which is two-isomorphic to $G$ and contains $F^{\prime}$ as a subgraph. We may thus assume that $F$ and $G$ are both twoconnected.

Now assume $\left\{v, v^{\prime}\right\}$ is a two element cutset of $F$ separating $F$ into vertex components $F_{1}$ and $F_{2}$. Let $G_{i}$ be all the vertices path connected to a vertex in $F_{i}-\left\{v, v^{\prime}\right\}$ by a path disjoint from $v$ and $v^{\prime}$. Then no vertex in $G$ is path connected to a vertex in $G_{2}$ by a path disjoint from $v$ and $v^{\prime}$ since by (4.3.2) this would guarantee an edge $e$ in $F$ which connects $F_{1}-$ $\left\{v, v^{\prime}\right\}$ to $F_{2}-\left\{v, v^{\prime}\right\}$. Hence $\left\{v, v^{\prime}\right\}$ is a cutset in $G$ and any (**) operation of $F$ can be extended to $G$.

ALTERNATE PROOF. We mention another proof as an illustration of some of our previous theorems. We will show as above that if $\left\{v, v^{\prime}\right\}$ is a cutset of $F$, it is a cutset of $G$ and the rest of the proof will follow as above. But if $\left\{v, v^{\prime}\right\}$ is a cutset of $F$ then adjoining the edge $e$ to $F$ between $v$ and $v^{\prime}$ if necessary (in which case $F \cup e$ is still a modular flat by 5.7) and then contracting the edge $e,[e, F \cup e]$ remains modular by (3.9) but has as a cutpoint the identified vertex $\overline{v v^{\prime}}$. But (3.16) guarantees $\overline{v v^{\prime}}$ is a cutpoint of $[e, G \cup e]$ so that $\left\{v, v^{\prime}\right\}$ is a cutset of $G$.

REMARKS 6.11. The alternate proof above suggests an extension to geometries of the graphical concept of multiple connectedness. If we define the connectedness of a geometry $c(G)$ recursively by $c(G)=0$, if $G$ is separable or of rank one while otherwise

$$
c(G)=\min _{p, q}\{c([p, G \cup p]), c([q, G])\}
$$


over all $q \in G$ and single element extensions of $G$ by $p$ we note that among graphical geometries representable by (connected) graphs, then $c(G)=k$ iff $G$ is graphically $(k+1)$-connected. We also have (by modifying (6.10) and using (3.9) and (5.7)) that if $G$ has a modular flat $F$ of rank $n$ and connectedness $k<n-1$, then $G$ has connectedness at most $k$.

THEOREM 6.12. Let $G$ and $H$ be geometries in a chain group $K$. Let $x$ be a modular flat of $G$, a subgeometry of $H$, and have the extension property in either $G$ or $H(6.1)$. Hence we may assume

$$
\begin{aligned}
& M_{x} \text { represents } x, \\
& M=\left[\begin{array}{ll}
M_{x} & M_{1} \\
0 & M_{2}
\end{array}\right] \text { represents } G, \text { and } \\
& M^{\prime}=\left[\begin{array}{ll}
M_{x} & M_{1}^{\prime} \\
0 & M_{2}^{\prime}
\end{array}\right] \text { represents } H .
\end{aligned}
$$

Then the generalized parallel connection $P_{x}(G, H)$ is in $K$ with the representation

$$
M(P)=\left[\begin{array}{ccc}
M_{2} & 0 & 0 \\
M_{1} & M_{x} & M_{1}^{\prime} \\
0 & 0 & M_{2}^{\prime}
\end{array}\right]
$$

Proof. Clearly $M(P)$ exhibits both $G$ and $H$ as subgeometries with $x$ as a modular flat of $G$. But contracting by $\bar{M}_{x}$ we have the interval $\left[\bar{M}_{x}, M(P)\right]$ represented by the direct sum

$$
\left[\begin{array}{ll}
M_{2} & 0 \\
0 & M_{2}^{\prime}
\end{array}\right]
$$

(with any zero column and any column which is a scalar multiple of another column deleted) which represents $\left[M_{x}, M\right] \oplus\left[\bar{M}_{x}, M^{\prime}\right]$. We are then done by (5.9).

COROLlARY 6.13. If $G$ and $H$ are binary (respectively ternary) pregeometries, so is $P_{x}(G, H)$ which is represented as in (6.12).

Proof. We apply (6.6) to (6.12).

COROLLARY 6.14. If $G$ and $H$ are in the chain group $K$ and $p$ is a point forming the intersection of $G$ and $H$, then the parallel connection $P_{p}(G, H)$ is also in $K$. 
Proof. We apply (6.7) to (6.12).

We remark that Conjecture 6.8, if true, would guarantee that any chain group would be closed under the taking of generalized parallel connections. A consequence of (6.5) is that if $x$ is binary, while $G$ and $H$ are in the chain group $K$, so is $P_{x}(G, H)$.

THEOREM 6.15. If $G$ and $H$ are two unimodular geometries with $x$ a modular flat of $G$ and a subgeometry of $H$ where using (6.6) we may assume $G$ is represented by the totally unimodular matrix

$$
M=\left[\begin{array}{lll}
M_{x} & 0 & M_{1} \\
0 & I & M_{2}
\end{array}\right]
$$

and $H$ by

$$
M^{\prime}=\left[\begin{array}{lll}
M_{x} & 0 & M_{1}^{\prime} \\
0 & I & M_{2}^{\prime}
\end{array}\right],
$$

then the matrix

$$
M(P)=\left[\begin{array}{ccccc}
M_{2} & I & 0 & 0 & 0 \\
M & 0 & M_{x} & 0 & M_{1}^{\prime} \\
0 & 0 & 0 & I & M_{2}^{\prime}
\end{array}\right]
$$

is totally unimodular and represents $P_{x}(G, H)$.

Proof. That $M(P)$ represents $P_{x}(G, H)$ over any field follows from (6.12). That it is totally unimodular then follows since if any nonzero subdeterminant $D$ were divisible by a prime $p$ (but not by a prime $q$ ) then the column vectors $C$ which determine $D$ along with a maximal subset of basic column vectors independent from $C$ would be independent over $G F(q)$ and dependent over $G F(p)$ contradicting the fact that $M(P)$ represents $P_{x}(G, H)$ over any field.

Research Problem 6.16. A very important problem in the theory of integer programming is the characterization of all totally unimodular matrices $U$ over the integers. It is well known that examples of such matrices include the signed circuit incidence matrix of a directed graph (matrix of tensions) and its dual (matrix of flows) [7], [24]. This class is closed under row-echelon operations (4.2.2), orthogonality (matroid duality), and generalized parallel connections (6.15).

For example, if we take the generalized parallel connection of the matrix of flows of $K_{5}$ and the matrix of tensions of $K_{3,3}$ relative to a common 
three point line (which is a modular flat in both geometries by 3.15 ) we get a direct-sum irreducible totally unimodular 6 by 16 matrix which is neither graphic nor cographic (i.e. cannot be realized as either the matrix of tensions or flows of a graph).

Is the class $U^{\prime}$ consisting of all graphic and cographic matrices and closed under duals, generalized parallel connections, and row-echelon operations identical with $U$ ?

THEOREM 6.17. Assume $G$ and $H$ are two graphical geometries with $x$ a modular flat of $G$ and a subgeometry (subgraph) of $H$ (where using (6.10) we may assume $x$ as a subgraph of $G$ is graphically isomorphic to $x$ as a subgraph of $H)$. Then $P_{x}(G, H)$ is graphical, being representable by the graph $P$ formed from the (vertex and edge) identification of the subgraph $x$ of $G$ with the subgraph $x$ of $H$. Hence, the vertex set of $P, V(P)$, equals $(V(G)-$ $V(x)) \cup(V(H)-V(x)) \cup V(x)$ and two vertices are adjacent in $P$ iff they were in $G$ or $H$.

Proof. $G$ and $H$ are subgraphs and hence subgeometries of $P$ with the subgraph $x$ a modular flat of $G$. Further, contraction of $x$ corresponds graphically to identifying the vertex set $V(x)$ to a common vertex $\bar{v}$ (and removing loops and multiple edges). This operation makes $\bar{v}$ a cutpoint, oneconnecting the graph $G$ with $V(x)$ identified to $\bar{v}$ and $H$ with $V(x)$ identified to $H$. We are then done by (5.9).

EXAMPLES 6.18. The geometry $K_{4}$, the geometry of contractions of the complete four-graph, is representable as an affine geometry by the picture:

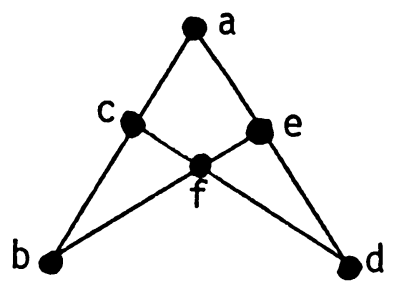

where $x=\{a, b, c\}$ is a modular flat. It is also representable by the totally unimodular matrix:

$$
\begin{aligned}
& \begin{array}{llllll}
a & b & c & d & e & f
\end{array} \\
& {\left[\begin{array}{llllll}
1 & 0 & 1 & 0 & 1 & 1 \\
0 & 1 & 1 & 0 & 0 & 1 \\
0 & 0 & 0 & 1 & 1 & 1
\end{array}\right]}
\end{aligned}
$$


and by the graph:

(3)

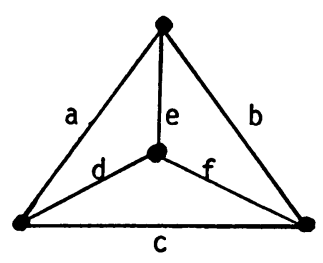

The geometry $P_{x}\left(K_{4}, K_{4}\right)$ can be represented in Euclidean three space by:

(4)

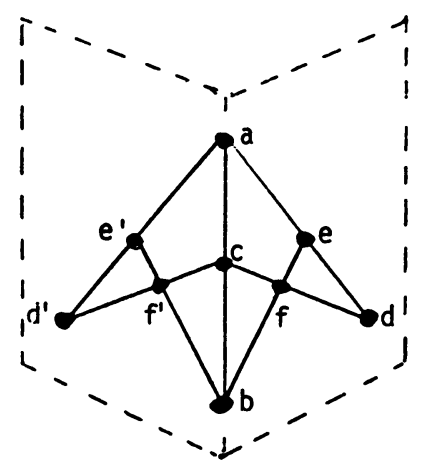

(the three-dimensional Desargues configuration), by the totally unimodular matrix:

(5)

$$
\begin{aligned}
& d^{\prime} e^{\prime} f^{\prime} \quad a \quad b \quad c \quad d e f \\
& {\left[\begin{array}{lllllllll}
1 & 1 & 1 & 0 & 0 & 0 & 0 & 0 & 0 \\
0 & 1 & 1 & 1 & 0 & 1 & 0 & 1 & 1 \\
0 & 0 & 1 & 0 & 1 & 1 & 0 & 0 & 1 \\
0 & 0 & 0 & 0 & 0 & 0 & 1 & 1 & 1
\end{array}\right]}
\end{aligned}
$$

and by the (planar) graph:

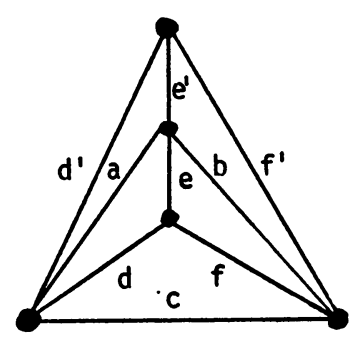

(7) However, graphical planarity is not preserved since the graph $G^{\prime}=$ $P_{x}\left(K_{4}, P_{x}\left(K_{4}, K_{4}\right)\right)$, although the generalized parallel connection of two planar graphs, is not planar since the subgraph $G^{\prime}-x$ is the (nonplanar) complete bipartite graph $K_{3,3}$.

(8) The class of transversal geometries is also not closed under parallel 
connections since if $G$ is the transversal geometry pictured (4.1.3) and $H$ is a three point line $\{c, x, y\}$ then $P_{c}(G, H)$ is not transversal since it has rank four but no four element subset spans all its cyclic flats [10]. We remark that Bixby had already noted that transversal pregeometries were not closed under parallel connections [4].

7. The characteristic polynomial, modularity, and the critical problem. In this section we show how the existence of a modular flat $x$ induces a factorization of the characteristic polynomial of a geometry $G: \chi(G, \lambda)=$ $\chi(x, \lambda) p(\lambda)$, a result proved by Stanley in [22]. We give a geometric interpretation of the quotient $p(\lambda)$ and then study the behavior of the characteristic polynomial and its associated invariants-the Möbius function [20] and Crapo's $\beta$ invariant [12] - under Brown truncation and generalized parallel connection. For appropriate classes of geometries these results can then be applied to the critical exponent of a geometry for chain groups and the chromatic number for graphs.

Definition 7.1. The Möbius function of a (geometric) lattice is defined recursively for all flats $x$ and $y$ of $G$ by $\mu(x, y)=0$ if $y \neq x, \mu(x, x)=1$, and $\mu(x, y)=-\Sigma_{x \leqslant z<y} \mu(x, z)$ if $y>x$. We then define $\mu(G)$ by $\mu(\varnothing, G)$ $=\mu(0,1)$ in the geometric lattice. The characteristic polynomial of $G, \chi(G)=$ $\chi(G, \lambda)$, equals the sum over all flats $y$ of $G: \Sigma \mu(0, y) \lambda^{r(G)-r(y)}$.

Properties of the characteristic polynomial [5] include $\chi(G, 0)=\mu(G)$, $\chi(G, 1)=0$ (so that $\chi(G)$ is divisible by $\lambda-1), \chi(G)=\chi(G-p) \chi(p)=$ $\chi(G-p)(\lambda-1)$ if $p$ is an isthmus of $G$, and $\chi(G)=\chi(G-p)-\chi([p, G])$ if $p$ is not an isthmus of $G$.

The Crapo $\beta$ invariant is given by

$$
\beta(G)=(-1)^{r(G)} \sum_{x \in G} \mu(0, x) r(x)=\left|\frac{d \chi}{d \lambda}\right|_{\lambda=1}|=| \chi^{\prime}(G, 1) \mid .
$$

Proposition 7.2 (StAnley). If $x$ is a modular flat of $G$ then the characteristic polynomial of $x, \chi(x)$, divides $\chi(G)$.

Proof. We use induction on the number of points contained in $G$ but not $x,|G-x|$. If $|G-x|=\varnothing$ then $G=x$ and hence $\chi(G)=\chi(x)$. Assume the proposition holds for all $G^{\prime}$ and $x$ such that $\left|G^{\prime}-x\right|<k$ and let $|G-x|=k+1$ with $p$ in $G-x$. If $p$ is an isthmus of $G$ then $\chi(G)=$ $\chi(G-p)(\lambda-1)$. Otherwise $\chi(G)=\chi(G-p)-\chi([p, G])$. But by (3.9), $x$ in $G$ is isomorphic to the modular flat $x$ in $G-p$ and the modular flat $[p, x \vee p]$ in $[p, G]$ respectively. But $|[p, G]-[p, x \vee p]| \leqslant|(G-p)-x|$ $=k$ so that by induction $\chi(x)$ divides $\chi(G-p)$ and $\chi([p, G])$ so it divides their difference as well as $\chi(G-p)(\lambda-1)$. 
THEOREM 7.3. 1. If $T(G)$ represents the upper truncation of the geometry $G$, then

$$
\chi(T(G))=\sum_{y \in G ; r(y)<r(G)-1} \mu(0, y)\left(\lambda^{r(G)-r(y)-1}-1\right) .
$$

Hence if $\chi(G)=\Sigma_{k=0}^{n} c_{k} \lambda^{k}$ then

$$
\chi(T(G))=\sum_{k=1}^{n} c_{k} \lambda^{k-1}+c_{0} .
$$

2. If $x$ is a modular flat with $r(x) \geqslant 2$, and if $T_{x}(G)$ represents the Brown truncation of $G$ relative to $x$ (5.12), then

$$
\chi\left(T_{x}(G)\right)=\chi(G) \chi(T(x)) / \chi(x)
$$

where $\chi(T(x))$ can be computed as above.

Proof. Since $T(G)$ has rank one less than $G$ and since $G$ and $T(G)$ agree up to level $r(G)-2$, the coefficient of $\lambda^{k}$ in $\chi(G)$ and the coefficient of $\lambda^{k-1}$ in $\chi(T(G))$ agree for all $1<k \leqslant r(G)$ while the constant term of $\chi(T(G))$ may be computed by the equations $\chi(T(G), 1)=\chi(G, 1)=0$.

To prove the second statement we use induction on $|G-x|$ noting that if $|G-x|=0$, then $G=x, T_{x}(G)=T(G)$, and $\chi\left(T_{x}(G)\right)=\chi(G) \chi(T(G)) / \chi(G)$. Assume the theorem holds for $G^{\prime}$ and $x$ such that $\left|G^{\prime}-x\right| \leqslant k$ and let $|G-x|=k+1$ with $p$ in $G-x$. Then using the arguments of (5.14), (7.1), and the induction hypothesis as in the proof of (7.2) we have if $p$ is an isthmus of $G$, it is an isthmus of $T_{x}(G)$ in which case

$$
\chi\left(T_{x}(G)\right)=\chi\left(T_{x}(G-p)\right)(\lambda-1)=\frac{\chi(G-p) \chi(T(x))}{\chi(x)}(\lambda-1)=\frac{\chi(G) \chi(T(x))}{\chi(x)}
$$

while if $p$ is not an isthmus of $G$ it is also not an isthmus of $T_{x}(G)$ and we have

$$
\begin{aligned}
\chi\left(T_{x}(G)\right) & =\chi\left(T_{x}(G)-p\right)-\chi\left(\left[p, T_{x}(G)\right]\right)=\chi\left(T_{x}(G-p)\right)-\chi\left(T_{[p, x \vee p]}([p, G])\right) \\
& =\frac{\chi(G-p) \chi(T(x))}{\chi(x)}-\frac{\chi([p, G]) \chi(T([p, x \vee p]))}{\chi[p x \vee p]} \\
& =\frac{(\chi(G-p)-\chi([p, G])) \chi(T(x))}{\chi(x)}=\frac{\chi(G, \chi(T(x))}{\chi(x)} .
\end{aligned}
$$

We now give an explicit geometrical description of the polynomial obtained when $\chi(G)$ is divided by $\chi(x)$. 
COROLLARY 7.4. If $x$ is a modular flat of $G$, then the complete Brown truncation has the characteristic polynomial $\chi\left(\bar{T}_{x}(G)\right)=\chi(G)(\lambda-1) / \chi(x)$. Hence the Stanley quotient $\chi(G) / \chi(x)$ is equal to the characteristic polynomial of the geometry formed from the flats of $G$ containing $x$ and those disjoint from $x$, divided by $\lambda-1$.

Proof. If $r(x)=n$, we apply (7.3.2) $n-1$ times obtaining:

$$
\begin{aligned}
\chi\left(T_{x}^{n-1}(G)\right) & =\chi\left(T_{T^{n-2}(x)}\left(T_{T^{n-3}(x)}\left(\cdots\left(T_{x}(G)\right) \cdots\right)\right)\right) \\
& =\frac{\chi\left(T^{n-1}(x)\right)}{\chi\left(T^{n-2}(x)\right)} \cdot \frac{\chi\left(T^{n-2}(x)\right)}{\chi\left(T^{n-3}(x)\right)} \cdots \frac{\chi(T(x))}{\chi(x)} \cdot \chi(G)=\frac{\chi\left(T^{n-1}(x)\right) \chi(G)}{\chi(x)} .
\end{aligned}
$$

But $\chi\left(T^{n-1}(x)\right)=\lambda-1$ since $T^{n-1}(x)$ is a rank one geometry.

COROLlaRy 7.5 (STANLeY). If $x$ is a modular copoint of $G$ then $\chi(G)=\chi(x)(\lambda-k)$ where $k=|G-x|$.

Proof. By (7.4), $\chi(G)=\chi\left(\bar{T}_{x}(G)\right) \chi(x) /(\lambda-1)$. But since $x$ is a modular copoint, $y$ is disjoint from $x$ iff $y=p \in G-x$. Hence $\bar{T}_{x}(G)$ is isomorphic to $L_{k+1}$, the rank two geometry (line) with $k+1=|G-x|+1$ points, and one readily checks that $\chi\left(L_{k+1}\right)=\lambda^{2}-(k+1) \lambda+k=(\lambda-k)(\lambda-1)$.

COROLlARY 7.6 (STANLEY). A supersolvable geometry is one with a saturated chain $\left\{x_{0}=\varnothing, x_{1}, \cdots, x_{n-1}, x_{n}=G\right\}$ of modular flats. Such geometries were introduced by Stanley in [22] and [23]. They form a class closed under contractions, deletions by points in $G-x_{n-1}$, and direct sums; and the class contains, for example, the partition lattices, projective geometries, rank one and rank two geometries, and the Dowling lattices.

If $G$ is supersolvable, then $\chi(G)=\prod_{i=1}^{n}\left(\lambda-\left|x_{i}-x_{i-1}\right|\right)$.

Proof. This follows from (7.5) and the fact that $\left\{x_{0}, \cdots, x_{k}\right\}$ is a saturated chain in the (sub)geometry $x_{k}$.

Lemma 7.7. If $P=P_{x}(G, H)$ denotes the generalized parallel connection of $G$ and $H$ relative to $x$ (which is modular in $P$ ) then $\bar{T}_{x}(G) \simeq \bar{T}_{H}(P)$.

Proof. $H$ is a modular flat of $P$ by (5.10.1). We then note that $y$ is a flat of $P$ containing $H$ iff $y \cap G$ is a flat of $G$ containing $x$ and that $y^{\prime}$ is a flat of $P$ disjoint from $H$ iff $y^{\prime}$ is a flat of $G$ disjoint from $x(5.2,5.3)$. We are then done by (5.14.3).

THEOREM 7.8. The characteristic polynomial of the generalized parallel connection may be computed by $\chi\left(P_{x}(G, H)\right)=\chi(G) \chi(H) / \chi(x)$.

PROoF. Using (7.4) and (7.7) we obtain 


$$
\chi(P)=\frac{\chi(H) \chi\left(\bar{T}_{H}(P)\right)}{\lambda-1}=\frac{\chi(H)}{\lambda-1} \cdot \chi\left(\bar{T}_{x}(G)\right)=\frac{\chi(H)}{\lambda-1} \frac{\chi(G)(\lambda-1)}{\chi(x)} .
$$

Proposition 7.9. If $x$ is a modular flat of $G$ with characteristic polynomial $\chi(x, \lambda)=\Sigma_{k=1}^{n} c_{k} \lambda^{k}$, then for $r(x)>1$,

$$
\mu\left(T_{x}(G)\right)=\frac{\mu(G) \mu(T(x))}{\mu(x)}=\frac{\mu(G)\left(c_{1}+c_{0}\right)}{c_{0}} .
$$

Further

$$
\mu\left(\bar{T}_{x}(G)\right)=\frac{-\mu(G)}{\mu(x)} \text { and } \mu\left(P_{x}(G, H)\right)=\frac{\mu(G) \mu(H)}{\mu(x)},
$$

and if $x$ is connected then

$$
\begin{gathered}
\beta\left(T_{x}(G)\right)=\frac{\beta(G) \beta(T(x))}{\beta(x)} \quad(r(x)>1) ; \\
\beta\left(\bar{T}_{x}(G)\right)=\frac{\beta(G)}{\beta(x)} ; \quad \text { and } \beta\left(P_{x}(G, H)\right)=\frac{\beta(G) \beta(H)}{\beta(x)} .
\end{gathered}
$$

Proof. The equalities involving the Mobius function are evaluations at $\lambda=0$ of the equations in (7.3.2), (7.4), and (7.8) respectively.

The formulas involving $\beta$ are the results of differentiating the equations in (7.3.2), (7.4), and (7.8) and then evaluating at $\lambda=1$, noting that

$$
\left.\left|\frac{\chi(G)}{\chi(x)}\right|_{\lambda=1}\right|_{1}=\frac{\beta(G)}{\beta(x)}
$$

by L'Hôpital's rule since the latter exists when $x$ is connected. But if $g / f=$ $g^{\prime} / f^{\prime}$, then $(g h / f)^{\prime}=g^{\prime} h^{\prime} / f^{\prime}$.

DEFINITION 7.10. The critical exponent [16] of a geometry $G$ of rank $n$ in the chain group of geometries coordinatizable over a finite field $F$ is the minimum integer $c$ such that for an imbedding of $G$ into $P_{n}(F)$, the projective geometry of rank $n$ over $F$, there exist $c$ hyperplanes $H_{1}, \cdots, H_{c}$ such that $H_{1} \cap \cdots \cap H_{c} \cap F=\varnothing$. This number is independent of the imbedding and is also equal to the minimum number of linear functionals $f_{1}, \cdots, f_{c}$ in $V_{n}^{*}(F)$ such that for all points $p \in G$, there is an $f_{i}$ such that $f_{i}(p) \neq 0$.

We also have that $\chi\left(G,|F|^{k}\right)=0$ for $k=0,1, \cdots c-1$ and $\chi\left(G,|F|^{k}\right)>0$ for $k=c, c+1, c+2, \cdots$. This was shown in [16] and [7] where it was further proved that if $H$ is a subgeometry of $G$, then the critical exponent of $H$ is at most $c$.

Theorem 7.11. Assume $x$ is a modular flat of a geometry G. Then:

1. If $T_{x}(G)$ and $G$ are both coordinatizable over the finite field $F$ and 
$r(x)>1$, then the critical exponent of $T_{x}(G)$ is at most that of the maximum of the critical exponents of $G$ and $T(x)$.

2. If $\bar{T}_{x}(G)$ and $G$ are both coordinatizable, then the critical exponent of $\bar{T}_{x}(G)$ is less than or equal to the critical exponent of $G$.

3. If $P_{x}(G, H)$ is coordinatizable over $F$, then its critical exponent is equal to the maximum of the critical exponents of $G$ and $H$.

PRoof. The first two stantements follow from (7.3.2) and (7.4) since if $\chi\left(G,|F|^{c_{1}}\right)>0$ and $\chi\left(T(x),|F|^{c}\right)>0$ (where $T(x)$ is coordinatizable since it is a subgeometry of $\left.T_{x}(G)\right)$ then $\chi\left(x,|F|^{c_{1}}\right)>0$ by (7.10) while $|F|^{c_{1}}$ $1>0$ so that $\chi\left(T_{x}(G),|F|^{\max \left(c_{1}, c_{2}\right)}\right)>0$ and $\chi\left(\bar{T}_{x}(G),|F|^{c_{1}}\right)>0$.

Similarly we have if $\chi\left(G,|F|^{c_{1}}\right)>0$ and $\chi\left(H,|F|^{c_{2}}\right)>0$ then $\chi\left(x,|F|^{c_{1}}\right)>0$ by $(7.10)$ so that $\chi\left(P_{x}(G, H),|F|^{\max \left(c_{1}, c_{2}\right)}\right)>0$. Conversely, the critical exponent of $P_{x}(G, H)$ is at least as large as the critical exponent of either of its subgeometries $G$ and $H$ by (7.10).

DEFINITION 7.12. If $G$ is a graph then the chromatic number of $G$, $c(G)$, is the minimum number of colors needed to color the vertices of $G$ such that no two adjacent vertices of $G$ are assigned the same color. More generally, the chromatic polynomial of $G, c(G, \lambda)$, is the number of ways we may assign colors to the vertices of $G$ subject to the above restriction. Then $c(G, \lambda)=$ $\lambda^{k} \chi(G, \lambda)$ (where $k$ is the number of components of $G$ ) and $c(G)$ is the minimum positive integer such that $\chi(G, c)>0$ [7]. Again, if $H$ is a subgraph of $G$, then $c(H) \leqslant c(G)$.

The proof of the following theorem mimics that of (7.11).

THEOREM 7.13. If $G$ and $H$ are connected graphs, then the chromatic polynomial of their generalized parallel connection, $\bar{c}\left(P_{x}(G, H), \lambda\right)$, is equal to $\bar{c}(G, \lambda) \bar{c}(H, \lambda) / \bar{c}(x, \lambda)$, so that the chromatic number $c\left(P_{x}\right)$ of $P_{x}(G, H)$ is equal to $\max (c(G), c(H))$.

\section{BIBLIOGRAPHY}

1. G. Birkhoff, Lattice theory, 3rd ed., Amer. Math. Soc. Colloq. Publ., vol. 25, Amer. Math. Soc., Providence, R. I., 1967. MR 37 \#2638.

2. G. D. Birkhoff, A determinant formula for the number of ways of coloring a map, Ann. of Math. (2) 14 (1912), 42-46.

3. G. D. Birkhoff and D. C. Lewis, Chromatic polynomials, Trans. Amer. Math. Soc. 60 (1946), 355-451. MR 8, 284.

4. R. Bixby, Composition and decomposition of matroids and related topics, Thesis, Cornell University, Ithaca, N. Y., 1972.

5. T. Brown, Deriving closure relations with the exchange property. I, II (to appear).

6. T. Brylawski, A combinatorial model for series-parallel networks, Trans. Amer. Math. Soc. 154 (1971), 1-22. MR 44 \#5237. 
7. - A decomposition for combinatorial geometries, Trans. Amer. Math. Soc. 171 (1972), 235-282. MR 46 \#8869.

8. - The Möbius function on geometric lattices as a decomposition invariant, Proc. Conference on Möbius Algebras, Univ. of Waterloo, 1971, pp. 143-148.

9. - An outline for the study of combinatorial pregeometries, Notes, University of North Carolina, Raleigh, N. C., 1972.

10. - An affine representation for transversal geometries, Studies in Applied Mathematics (to appear).

11. T. Brylawski and D. Lucas, Uniquely representable combinatorial geometries, Proc. Internat. Colloq. on Combinatorial Theory, Rome, Italy, 1973.

12. H. Crapo, $A$ higher invariant for matroids, J. Combinatorial Theory 2 (1967), 406-417. MR 35 \#6579.

13. - The joining of exchange geometries, J. Math. Mech. 17 (1968), 837852. MR $39 \# 2657$.

14. Chromatic polynomials for a join of graphs, Combinatorial Theory and its Applications (Proc. Colloq., Batalonfüred, 1969), North-Holland, Amsterdam, 1970, pp. 239-245. MR 45 \#8572.

15. Constructions in combinatorial geometries, Notes, Bowdoin College, Brunswick, Me., 1971.

16. H. Crapo and G.-C. Rota, On the foundations of combinatorial theory: Combinatorial geometries, M. I. T. Press, Cambridge, Mass., 1970 (Preliminary edition). MR 45 \#74.

17. W. Graves, An algebra associated to a combinatorial geometry, Bull. Amer. Math. Soc. 77 (1971), 757-761. MR 44 \#3897.

18. C. Greene, $A$ rank inequality for finite geometric lattices, J. Combinatorial Theory 9 (1970), 357-364. MR 42 \#1727.

19. - On the Möbius algebra of a partially ordered set, Proc. Conference on Möbius Algebras, Univ. of Waterloo, 1971, pp. 3-20.

20. G.-C. Rota, On the foundations of combinatorial theory. 1, Theory of Möbius functions, Z. Wahrscheinlichkeitstheorie und Verw. Gebiete 2 (1966), 340-368. MR 30 \#4688.

21. J. Sonner, The language of arrows (to appear).

22. R. P. Stanley, Modular elements of geometric lattices, Algebra Universalis 1 (1971/72), 214-217. MR 45 \#5037.

23. - Supersolvable semimodular lattices, Proc. Conference on Möbius Algebras, Univ. of Waterloo, 1971, pp. 80-142.

24. W. T. Tutte, Lectures on matroids, J. Res. Nat. Bur. Standards Sect. B 69B (1965), 1-47. MR 31 \#4023.

25. H. Whitney, 2-isomorphic graphs, Amer. J. Math. 55 (1933), 245-254.

26. L. R. Wilcox, Modularity in the theory of lattices, Ann. of Math. 40 (1939), 490-505.

27. T. A. Dowling and R. M. Wilson, Whitney number inequalities for geometric lattices, Proc. Amer. Math. Soc. 47 (1975), 504-512.

DEPARTMENT OF MATHEMATICS, UNIVERSITY OF NORTH CAROLINA, CHAPEL HILL, NORTH CAROLINA 27514 